\title{
Two-Dimensional Saccade-Related Population Activity in Superior Colliculus in Monkey
}

\author{
RUSSELL W. ANDERSON, EDWARD L. KELLER, NEERAJ J. GANDHI, AND SANJOY DAS \\ Smith-Kettlewell Eye Research Institute, San Francisco, 94115; and Graduate Group in Bioengineering, \\ University of California, San Francisco and University of California, Berkley, California 94720
}

\begin{abstract}
Anderson, Russell W., Edward L. Keller, Neeraj J. Gandhi, and Sanjoy Das. Two-dimensional saccade-related population activity in superior colliculus in monkey. J. Neurophysiol. 80: 798817,1998 . The two-dimensional distribution of population activity in the superior colliculus (SC) during saccadic eye movements in the monkey was estimated using radial basis functions. To make these ensemble activity estimates, cells in the deeper layers of the SC were recorded over much of the rostrocaudal (caudal to 3.8 $\mathrm{mm}$ from the rostral tip), mediolateral extent of this structure. The dynamic movement field of each cell was determined at 2-ms intervals around the time of saccades for a wide variety of horizontal and oblique movements. Collicular neurons were divided into partially overlapping dorsal and ventral cell layers on the basis of recorded depth in SC. The pattern of presaccadic activity was used as an additional discriminant to sort the cells in the two layers into separate burst (dorsal) and buildup (ventral) cell classes. Rostrocaudal and medioventral cell location on the colliculus was estimated from the optimal target vector for a cell's visual response rather than from the optimal motor vector. The former technique was more reliable for locating some buildup neurons because it produced locations that compared better with the locations suggested by electrical stimulation. From the movement field data and from the estimates of each cell's anatomic location, a similar algorithm was used to compute the two-dimensional population activity in the two layers of the SC during horizontal and oblique saccades. A subset of the sample of neurons, located near the horizontal meridian of the SC, first was used to compute onedimensional dynamic population activity estimates for horizontal saccades to allow partial comparison to previous studies. Statistical analyses on the one-dimensional data were limited to saccades of $\leq 20^{\circ}$. The analyses indicated that while there was a small rostrally directed shift in the center of gravity of the distributed activity in the buildup cell layer, there was little support for the theory of a systematic rostrally directed spread of the leading edge of the activity. The two-dimensional results extend the previous one-dimensional estimates of collicular activity during saccades. Discharge in the burst layer was invariant in size for all saccade vectors and symmetrically arranged about a center of gravity that did not move during saccades. The size of the active area in the buildup layer grew modestly with saccade amplitude, whereas the distribution of activity was skewed toward the rostral end of the SC for saccades larger than $10^{\circ}$. There was a small, but consistent shift in the center of gravity of the two-dimensional activity that was directed along the horizontal meridian (for horizontal movements) or an oblique meridian (for oblique movements) of the SC. However, the spread of activity during a saccade was as large or larger in the mediolateral direction as it was in the rostral direction. The results indicate that changes in activity occur in an extended zone on the SC, and in all directions but caudal, in the buildup layer during saccades and do not support the idea of a rostrally directed spread of activity as a dynamic control mechanism for saccades. Our results and those of previous investigators of collicular popula-
\end{abstract}

tion activity may be limited by stationarity concerns in that the cells used to estimate population activity were recorded in several monkeys over an extended period of time to obtain a sufficient spatial sample.

\section{INTRODUCTION}

The superior colliculus ( $\mathrm{SC}$ ) is a crucial participant in the neural control of saccadic eye movements (see review by Sparks and Hartwich-Young 1989), but its precise role in the generation of saccades remains controversial. It is generally agreed that the SC exerts its control function through population coding in which large ensembles of neurons in its deeper layers are active for every saccade. The location of the most intense activity is dependent on the size and direction of the movement in a topologically organized fashion over the surface of the SC, but precisely what the population activity codes remains unclear. According to one theory, the saccadic movement is based on a vector summation mechanism in which each member of the active population of collicular neurons contributes a minivector of movement control according to its topographical location on the collicular map (Van Gisbergen et al. 1987). In another view, the saccadic vector is determined by an averaging process that computes the center of mass of the active population (Lee et al. 1988; Van Opstal and Van Gisbergen 1989). In each of these hypotheses, the SC is viewed as providing the static goal (desired eye displacement), and the dynamics of the control process are carried out downstream in a local feedback network that continuously compares the desired eye displacement, as specified by the SC population code, to the actual current eye displacement during a saccade provided by an efferent (local) feedback mechanism (Robinson 1975).

Recent evidence suggests that SC activity also may play a role in dynamic control or at least be correlated with various dynamic saccadic signals (Berthoz et al. 1986; Das et al. 1995; Keller and Edelman 1994; Lee et al. 1988; Munoz et al. 1991a,b; Waitzman et al. 1991). A novel dynamic hypothesis supposing a rostrally spreading wave of activity on the SC during saccades also has been proposed (Munoz and Wurtz 1995b; Optican 1995; Wurtz 1996). The latter theory suggests that three functionally distinct types of neurons exist in the deeper layers of the SC: dorsally located burst neurons, more ventrally placed buildup neurons, and rostrally situated fixation neurons. Before saccade onset, a spatially extensive population of buildup cells begins to discharge at low frequency while fixation cells start to decrease their discharge. Shortly before saccade onset, burst neurons 
in a circumscribed region centered on the site of maximal activity in the buildup cell population begin a high-frequency burst of discharge that approximately coincides with saccade onset. As the movement proceeds, the buildup cells receive feedback about the ongoing change in eye position in the form of instantaneous eye velocity. The network of active buildup cells performs a spatial integration on the velocity signal, a process that generates a rostrally directed spread of activity in the buildup cell layer. The active burst cell population remains fixed at its initial collicular location and declines in firing rate as the spread of activity moves the center of activity in the buildup layer in a rostral direction. Finally, when the spread of activity in buildup cells reaches the rostral limit of the SC, it reactivates fixation neurons, a mechanism which ends the saccade. A somewhat similar concept had been proposed previously based on cellular recordings in the cat superior colliculus (Munoz et al. 1991b).

The goal of the present investigation was to compute the two-dimensional, spatiotemporal activity in the three types (according to the Munoz and Wurtz classification scheme) of collicular neurons during both oblique and horizontal saccades. We then use the two-dimensional results to evaluate the validity of the rostrally spreading wave hypothesis. Most previous studies (Munoz and Wurtz 1995b; Munoz et al. 1991a,b) have been limited to estimating population activity along the central meridian of the colliculus during horizontal saccades. In one exception, Ottes et al. (1986) presented a two-dimensional estimate of the shape and extent of the population activity for burst cells. Their estimate was based on an amplitude series (at best direction) and a direction series (at best amplitude) through the movement fields of cells at a limited set of locations on the collicular map. Their data had the further limitation that the mean firing rate during a $100-\mathrm{ms}$ time bin centered on the saccade onset was the measure of activity and hence cannot be used to test dynamic theories of saccadic control. A comprehensive examination of the spreading activity theory requires knowledge of the population activity over the entire colliculus because the motor map in this structure is known to represent all contralateral saccades including up and down oblique movements (Robinson 1972). Two-dimensional estimations are needed to examine the generality of the hypothesis when extended to oblique movements (Optican 1995). They also are needed to test an alternative hypothesis in which the activity of buildup neurons in a broad region surrounding the center of activity is inhibited before saccade onset and then comes back on near the end of the saccade but without a rostrally directed order of reactivation (Gandhi et al. 1994). In the latter theory, it is the decline of high-level activity at the center of the active region as the saccade progresses that codes movement dynamics.

We followed the general approach used by previous groups to estimate population activity from single-cell recordings (Munoz and Wurtz 1995b; Ottes et al. 1986). We first characterized the movement fields of individual neurons positioned as evenly as possible over the collicular surface. The movement field estimates for individual cells at closely spaced intervals of time then were used to compute the evolution of population activity on the colliculus for each of the cell types as the saccade progressed.

We found that none of the previous approaches to estimat- ing the movement fields of cells in two-dimensions could be used uniformly across all the cells in our sample. Nonlinear regression models such as bivariate Gaussian fits or logGaussian amplitude fits (Bruce and Goldberg 1985) did not converge for many cells, particularly those of the buildup class. Therefore we developed a new algorithm using radial basis functions (Moody and Darken 1989) that was computationally tractable and provided function independent estimations of the movement fields of all neurons. The details of the methodology for the application of this algorithm are described in a separate paper (Anderson et al. 1998). The present paper concentrates on applying the algorithm to the problem of specifying the evolution of the population activity in the SC during both horizontal and oblique saccades.

\section{METHODS}

\section{Surgical and physiological procedures}

Data presented in this report have been pooled from two Macaca mulatta and one M. fasicularis male, adolescent monkeys. All experimental protocols were approved by the Institute Animal Care and Use Committee at the California Pacific Medical Center and complied with the guidelines of the Public Health Service policy on Humane Care and Use of Laboratory Animals. The animals were prepared for the experiments in a single surgery under aseptic conditions. Anesthesia was induced with an intramuscular injection of ketamine $(15 \mathrm{mg} / \mathrm{kg})$ and acepromazine $(0.1 \mathrm{mg} / \mathrm{kg})$. The monkeys then were intubated and anesthetized with isoflurane for the duration of the surgical procedure. Heart rate, respiratory rate, pulse oximeter, and body temperature were monitored throughout the procedure. The following devices were implanted in the animal during the surgery: 1 ) a coil of Teflon-coated wire under the conjunctiva of one eye using the method of Judge et al. (1980); 2) a stainless steel recording chamber placed stereotactically on the skull, slanted posteriorly at an angle of $38^{\circ}$ in the sagittal plane and aligned on the SC. This angle ensured that recording penetrations were made approximately perpendicular to the surface of the SC. And 3) two stainless steel bars transversely above the skull anterior to the recording chamber for use as a head restraint device. After surgery, monkeys recovered rapidly from the gas anesthesia and then were returned to their home cage. The following day they received an injection of an analgesic agent (buprenex, $0.1 \mathrm{mg} / \mathrm{kg}$ im). They were allowed to recover completely from the effects of the surgery before behavioral training was begun.

Eye movements were recorded with the magnetic, search coil technique (Robinson 1963). Visual targets (bright spots of light of $1 / 4^{\circ}$ visual angle) were back-projected on a translucent tangent screen located at a distance of $35 \mathrm{~cm}$ from the animal's eyes with an oscilloscope projection system (Crandall and Keller 1985). The tangent screen was illuminated with a dim homogenous background $\left(0.1 \mathrm{~cd} / \mathrm{cm}^{2}\right)$ and the visual display subtended $\pm 45^{\circ}$ of visual angle along both the horizontal and vertical axes. The position of the targets, the behavioral paradigms, and the storage of data were controlled by a single, real-time program running on a PC computer. Horizontal and vertical eye velocity signals were produced by analog differentiation of the corresponding eye position record (cutoff frequency of $170 \mathrm{~Hz}$ ). Eye position, eye velocity, and target position signals were digitized at $1 \mathrm{kHz}$ and stored to disk.

Single neurons were recorded with tungsten microelectrodes (Frederick Haer) lowered through the chamber within a guide tube which penetrated the dura. The position of the electrode within the chamber was determined by a double-eccentric micro-device that allowed the microelectrode to access any position within the 12$\mathrm{mm}$ diameter chamber. 
The depth on the hydraulic drive when the microelectrode first entered the dorsal surface of the SC was noted. This boundary was distinguished clearly by the intense background activity generated in superficial layer neurons as the monkey made spontaneous saccades across a textured background. Once the dorsal surface of the SC had been determined, the electrode was advanced further into the colliculus while the monkey made visually guided saccades in response to target steps. At a depth $\sim 1.0 \mathrm{~mm}$ below the surface, the first visuomotor collicular cells were encountered, and we then started the animal on the behavioral paradigm (described later) and recorded every cell we could isolate as the electrode was advanced through the deeper layers of the colliculus. Single-cell discharges were detected by a window discriminator and were stored in register with the analog data at a resolution of $1 \mathrm{kHz}$.

In two of the animals, we delivered short trains of electrical stimulation through the recording electrode at the location in the deeper layers of SC where we had recorded the most ventrally located neuron on that penetration. Stimulation (range of parameters: $25-35 \mu \mathrm{A}, 25-75 \mathrm{~ms}, 400$ pulses/s) was applied by the computer after the animal had first obtained fixation on a visual target that then had been turned off for 100-200 ms. We recorded the fixed vector saccades that were evoked by this electrical stimulation.

\section{Behavioral paradigms}

The experimental paradigm used in the present experiment was a variation of a delayed saccade protocol. The monkey was required initially to fixate a stationary visual target. After a random interval ( selected uniformly from a continuum over 500-900 ms ), a second target spot appeared at an eccentric position while the fixation target remained on for an additional random interval (400-800 $\mathrm{ms}$, also from a continuum). After this second interval, the fixation target went off, which was the cue to the animal that it should now saccade to the location of the eccentric target. The second target was extinguished before the monkey could make a saccade to its location. We turned off the target to avoid any possible visual contribution to the saccadic response of units during large saccades, which could take $>100 \mathrm{~ms}$ to complete. The animal was rewarded for making accurate saccades to the former target location [a window size of $\pm 1^{\circ} \pm 0.1$ times the magnitude of the target displacement was placed around the location of the required saccade]. The lack of a visual target at the end of the saccades meant that corrective saccades were only very rarely observed. The fixation point was located at the straight-ahead position when the upcoming target displacement was to be $<20^{\circ}$ in amplitude. For target steps $>20^{\circ}$, the fixation point was offset from straight ahead to prevent restrictions due to the oculomotor range.

For each isolated cell, we determined a rough estimate of the center and extent of the cell's movement field by having the animal make saccades to manually chosen target locations while the operator examined on-line histogram displays of average unit discharge generated by the computer to aid the operator in qualitative, but immediate, assessment of a cell's discharge properties. A computer program then was activated that required the monkey to make saccades to a variety of positions chosen at random within and just beyond the borders of the field. When the field was very large and its outer borders could not be determined, the area over which saccades were tested was extended out to a maximum target displacement of $50^{\circ}$ in amplitude and $\pm 60^{\circ}$ in direction from the cell's preferred direction. The saccade vectors thus potentially covered a wide range of magnitude and direction in visual space, but because of the expansion of visual space with eccentricity, large saccades were not sampled as densely as smaller movements. The cell with the smallest sampling radius was $26^{\circ}$. Normally after the random set of saccades was obtained, additional data were collected for saccades made to locations near the center of the cell's move- ment field. In the data sets for some cells recorded in early experiments, targets only appeared at a fixed set of grid points rather than at random locations. Normally a data set for neurons with large field extent included $\sim 150$ saccades.

The animals were rewarded for correct responses with small drops of water. They worked until satiated each day during the week (usually 3-4 h) while single neurons were recorded. On weekends they received water ad lib in their home cages. Their weight was monitored every day and additional fruit or water was given as necessary.

\section{Data analysis}

The eye movement records initially were processed by a computer program to mark the beginning and end points of the saccades using eye velocity and acceleration criteria (Keller and Edelman 1994). Each record was examined by a human operator and improperly marked times were corrected by hand. Raw neural discharge was converted to spike density signals by convolving the spike trains with a Gaussian waveform ( $\sigma=4 \mathrm{~ms}$ ) (Richmond et al. 1987) to produce smooth continuous estimates of unit activity. Neural data then were aligned alternatively on the onset of the visual target, saccade onset or saccade end.

\section{Estimation of dynamic movement fields and population activity}

Movement field plots were constructed from the spike density data aligned on saccade end. Alignment on the end of each movement in the data set for individual cells is required to construct population activity estimates to test the hypothesis that a spread of activity is the dynamical process controlling the end of saccades.

Estimates of the two-dimensional movement field surfaces were generated using a radial basis function algorithm developed in our laboratory. The computational details of this algorithm are reported in a separate paper (Anderson et al. 1998) and are briefly outlined in this paper. The steps involved in estimation of the movement field surfaces are illustrated using the data set for a typical cell in Fig. 1. Figure $1 A$ shows the end point locations of 171 saccades included in this cell's database. From this data, the convex hull enclosing the eccentricity extrema for the saccades was determined. Then a preprocessing algorithm was applied to the entire data set to generate more regularly spaced data points. The data set was triangulated, and interpolated saccades were placed at the center of triangles in sparsely sampled regions (Fig. $1 B$ ). The spike density trains for these interpolated points were determined by bilinear interpolation of the discharge of the three nearest saccades at each point in time. A radial basis function network then was trained (optimized by a least-squares error criterion of the overall surface at the actual and interpolated data points) to compute a smooth surface fit (at each point in time) to the preprocessed data using an iterative training procedure. The optimization procedure essentially implements a Gaussian moving average with the size of the averaging window inversely proportional to the local data density. In regions of sparse data (including those with interpolating points), the spatial filter has a low frequency, allowing smoothing over the scattered data. In regions containing peaks of discharge, data density is increased, and the filter adapts to a higher spatial frequency band-pass, allowing the rapidly changing spatial data to be reproduced faithfully.

Movement field plots were obtained at 2-ms intervals from 150 $\mathrm{ms}$ before the end of saccades to $14 \mathrm{~ms}$ after their end for a total of 82 surface estimates per cell. One example for the illustrated cell at the time interval $40 \mathrm{~ms}$ before saccade end is shown in Fig. $1 C$. To test the hypothesis that systematic spread of activity in one class of collicular neurons controls saccade end, data used to esti- 

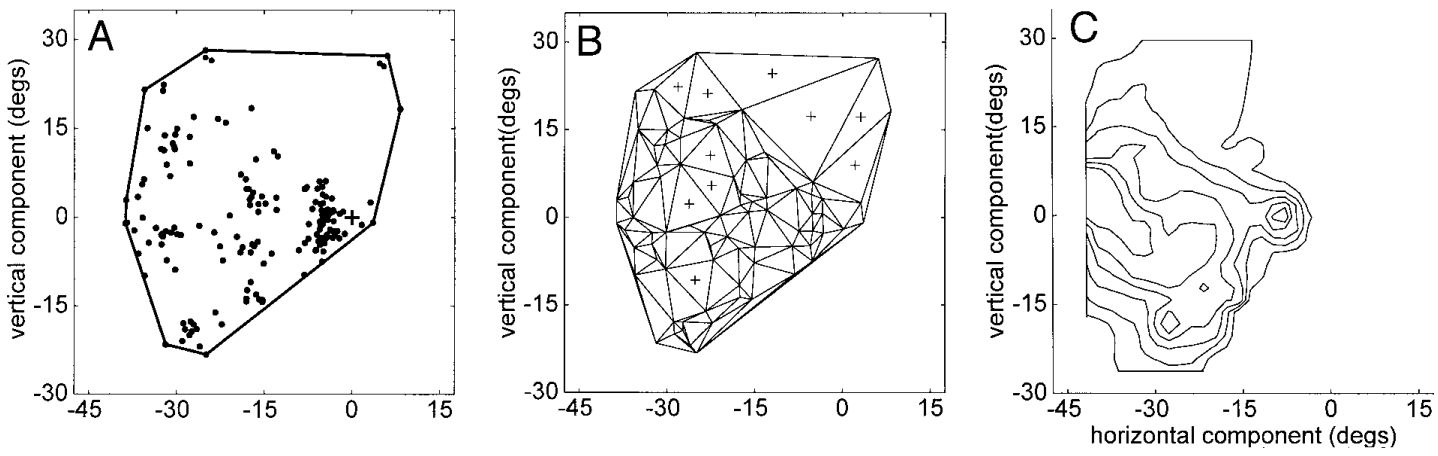

FIG. 1. Estimation of dynamic movement fields of collicular neurons using a radial basis function algorithm. $A$ : database for cell ba2201. $\bullet$, end point for 1 saccade in the total set $(n=171)$. Data shown in Cartesian coordinates with the abscissa being the horizontal component $(+$ is to right) and the ordinate the vertical coordinate $(+$ is up) of the movements. All saccades started near the fixation point shown as a cross at coordinate $(0,0)$. - , convex hull for the set of saccades. Note the limited amount of data in the top portion of the field. $B$ : data were preprocessed by triangulation. Where there were many closely spaced samples, the data were replaced by a single point at the apex of a computed triangle. Where the data were scarce, internal points were placed at the center of triangles above a threshold value of area $\left(50^{\circ}\right.$ squared in this example). $C$ : computed movement field of the neuron at a single time point $(40 \mathrm{~ms}$ before the end of all saccades in the set) shown as a contour plot. Contour lines are 150, 125, 100, 75, 50, and 25 spikes/s. Highest discharge is for nearly horizontal saccade of amplitude $40^{\circ}$. Because saccades of $40^{\circ}$ amplitude were the largest included in the data for this cell, the lefthand boundary of its field could not be determined (i.e., the cell was of open-field type).

mate the movement fields always were aligned on saccade end for all saccades in each cell's database.

The size (or extent) of a cell's movement field was quantified by the first and second moments of normalized two-dimensional movement field fits. The center of mass [in horizontal $(x)$ and vertical $(y)$ eye movement coordinates] of the movement field was specified by the first moment. The covariance matrix of the surface then was calculated. The square root of the eigenvalues of the covariance matrix defined the standard deviations (in degrees) of the activity along the major and minor axes of the field.

From the movement field plots for all cells and knowledge about their location on the colliculus (discussed in detail in RESULTS), we computed two-dimensional estimates of the spatiotemporal population activity over the surface of the colliculus contralateral to the saccades. For the two-dimensional analysis, a modification of our radial basis algorithm was used to compute the spatiotemporal distribution of the population activity in each layer as a function of collicular position [rostrolcaudal (u) and mediolateral (v)]. In a limited number of one-dimensional analyses of population activity for horizontal saccades, cubic smoothing splines were used to interpolate a smooth curve through the data (De Boor 1978). The smoothing parameter for the spline fits was fixed at 0.85 . These analyses were conducted to allow direct comparison to previously published one-dimensional work.

We tried to include saccades over a range of amplitudes from 1 to $40^{\circ}$ and over a range of directions $\pm 60^{\circ}$ about the preferred direction for each cell. For most cells, we obtained sufficiently placed saccadic data out to $38^{\circ}$ or even larger amplitudes to be able to plot the cell's movement field out to the limit of the available data. Nevertheless, on detailed off-line analysis we only obtained data out to $26^{\circ}$ in amplitude in eight cells, and we therefore limit our estimation of collicular population activity to saccades with amplitudes of $\leq 25^{\circ}$. This limitation arises for the population estimates because one must have movement field estimates for all cells in the population out to saccades at least as large as the modeled saccade. Thus the cell with the smallest, maximum eccentricity movement in its database determines the limit of the saccadic amplitude that can be modeled for population discharge. In other words, we can fit individual movement field estimates out to the limit of each cell's data but the population estimates are only valid for saccades smaller than the movement field data for the cell with the smallest extent of data $\left(26^{\circ}\right.$ in our sample of cells $)$. The center of mass (gravity) of the inverse fits was used also to quantify some of the dynamical properties of the population activity in the colliculus. The center of mass was defined as that point in twodimensional collicular space on which the spatial distribution of discharge (the population activity) would balance if the units of discharge were equivalent to mass.

\section{RES ULTS}

\section{Cell classification}

As the first step in the data analysis, we wanted to place each cell into one of the three classes, buildup neurons, burst neurons or fixation neurons, using the same criteria as those used by Munoz and Wurtz (1995a), so that we could compare our two-dimensional results to their one-dimensional experiments. We had statistical difficulty with the primary criterion used by these authors to divide cells outside the rostral pole of the SC (arbitrary defined as the most rostral $0.72 \mathrm{~mm}$ of colliculus ) into buildup or burst neurons. They classified cells located caudal to the rostral pole that discharged at a rate $>30$ spikes/s during the $100-\mathrm{ms}$ period of time ending $100 \mathrm{~ms}$ before saccadic onset (for movements of optimal size and direction in a delayed-saccade paradigm) as buildup neurons while those with discharge below this level as burst neurons. In Fig. 2, we plot the discharge for averaged optimal movements aligned on saccade onset for all 93 potential burst or buildup neurons from our sample. As the figure illustrates, collicular cells display a continuum of behavior with respect to the arbitrary criterion of 30 spikes/s for presaccadic discharge (Fig. 2B). Munoz and Wurtz (1995a) cited two additional criteria that they believed also served to separate cells in the deeper collicular layers into dynamically distinct classes (i.e., burst or buildup neurons ): depth from the dorsal surface of the colliculus and the spatial extent of the movement field (i.e., whether the cell displayed closed- or open-field discharge characteristics ). In our experience, these latter two criteria served as more definitive discriminates of cell type. We first placed 

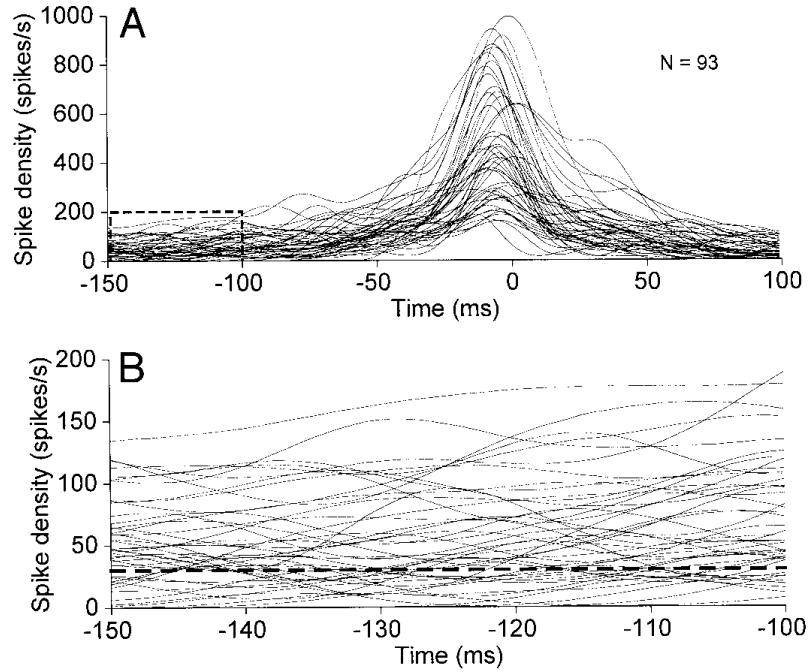

FIG. 2. Presaccadic discharge of neurons recorded in the deeper layers of the superior colliculus. $A$ : discharge for 93 cells in our sample recorded at various depths and positions on the superior colliculus (SC). Each trace shows the activity of 1 cell during the time period from $150 \mathrm{~ms}$ before to $100 \mathrm{~ms}$ after movement onset averaged for the 4 saccade trials in each cell's database for which the largest discharge occurred. Saccade onset is at 0 on this time scale. $B$ : blowup of the inset in $A$. Time scale now represents the 50-ms period ending $100 \mathrm{~ms}$ before saccade onset. - - - , criterion level of 30 spikes/s used by Munoz and Wurtz (1995a) to separate cells into burst or buildup classes.

cells recorded above a depth of $1.4 \mathrm{~mm}$ (and which had a saccade-related burst of activity) into the burst neuron class and those recorded below a depth of $1.6 \mathrm{~mm}$ into the buildup neuron classification. The total number of cells classified into the two types by depth alone was 72 . For the remaining 21 cells located between 1.4 and $1.6 \mathrm{~mm}$ in depth, we applied the 30 spikes/s criterion to determine classification. On some penetrations, cells were recorded at the border of the superficial layers and the intermediate layers with a sustained visual response that extended in time through the saccade. Because it was not possible to classify these cells as saccade related with our paradigm, they were dropped from further consideration, i.e., a cell in the region $>1.4 \mathrm{~mm}$ had to have a clear saccade-related burst to be included in the present analysis.

All cells that were classified as buildup neurons at any depth were required to have open-field discharge characteristics for the largest saccadic displacements (in the optimal direction) that were contained in the cell's data set. Otherwise the cell was dropped from further consideration for the purposes of this paper. Eight cells were dropped from the analysis on this basis. All the cells we include in the deeper layer sample for the population estimates were still discharging for saccades at least as large as $26^{\circ}$ (many were tested for larger movements), and the largest saccade for which we estimate population activity in the present paper was $25^{\circ}$. It is possible that for larger saccades some of our cells classified as buildup neurons may have closed fields.

To further specify the dynamic characteristics of the two types of cells classified by our criteria, we computed the average population discharge for each type of cell at a time point $75 \mathrm{~ms}$ before saccade onset. The average discharge for the deeper cells in our sample (hereafter called buildup cells) was $79.6 \pm 6.1$ spikes/s (mean $\pm \mathrm{SE}$ ) while the same measure for the more shallow cells (hereafter called burst neurons) was $39.1 \pm 9.2$ spikes/s. These average discharge rates were significantly different ( $t$-test, $P<0.01)$. The reason we picked the epoch $75 \mathrm{~ms}$ before saccade onset was that the average low-level presaccadic discharge in our two groups of cells had the maximum difference at this time. Nevertheless, there was still overlap in presaccadic discharge of individual members of our two classes of cells even at the $75 \mathrm{~ms}$ epoch.

Munoz and Wurtz (1995a) classified cells located in the rostral $0.72 \mathrm{~mm}$ of the buildup layer in the SC and those that paused for saccades, as fixation neurons. Neurons located more caudal but in the same layer of the SC were classified as buildup neurons. In our sample, deeper cells recorded in the rostral colliculus appeared to form a continuum with respect to their discharge properties between fixation and buildup neurons. Examples of this continuum in behavior are shown in Fig. 3. The cell shown on the left was recorded very rostral in the SC and paused for almost all saccades. However, when a saccade of 1.5 and $\sim 10^{\circ}$ up was made, the cell discharged a weak burst of activity (Fig. 3 , top ). But even for a slightly larger movement of $3^{\circ}$, the cell showed a dip in its activity for the movement (not shown ). For $20^{\circ}$ saccades, the cell was inhibited completely (Fig. 3, bottom). On the basis of its preferred saccade vector, this cell would be placed rostral to the $0.72 \mathrm{~mm}$ dividing
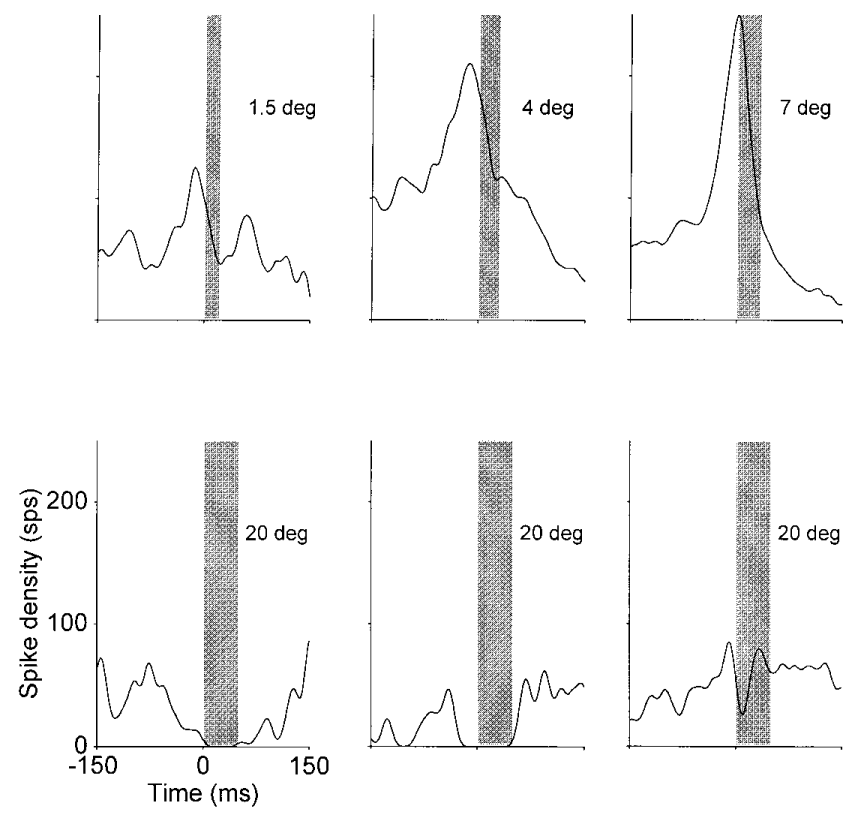

FIG. 3. Continuum of behavior in rostral, deep collicular cells. Each column of plots shows the activity of an individual cell. Top: average spike density for the cell for saccades of nearly optimal vector for that cell. Bottom: average density for a $20^{\circ}$ saccade in the same direction as the optimal vector. Average density traces computed from $\geq 4$ movements for all panels (except top left where only 2 saccades small enough and in the right direction were available to compute the average). Shaded vertical bar in each plot shows the average time duration of the saccadic eye movements associated with the discharge behavior in each plot. Both eye movements and neural discharge aligned on saccade onset (at 0 on each abscissa). Left: cell located in the rostral $0.5 \mathrm{~mm}$ of the colliculus. Middle: cell located at a region on the colliculus preferring $4^{\circ}$ movements. Right: cell located at a region on the colliculus preferring $7^{\circ}$ movements. Spike density and time calibrations (bottom left) apply to all panels. 
line and would be classified as a fixation neuron by the Munoz and Wurtz criterion. However, the cells shown in Fig. 3, right and middle, have similar behavior. Each has a preferred saccadic vector for which the cell bursts but then is inhibited for larger saccades in the same direction. The middle cell has a preferred magnitude of $4^{\circ}$, whereas the cell on the right prefers saccades of $7^{\circ}$ (placing both cells more caudal than the Munoz and Wurtz dividing meridian on the SC). These cells would be called buildup neurons by their criterion. However, it seems clear that both types have similar levels of early presaccadic discharge but then show a decline in their activity and finally an inhibition in activity for all contralateral saccades larger than a certain size. The saccade size limit required to produce the down trend and the pause in activity becomes progressively smaller as the recording location is moved more rostral on the SC. For saccades smaller than its limit, a particular cell shows an accelerating trend in discharge and a burst (for some cells very weak) of activity during the saccade. We also recorded another cell in which pauses occurred for saccades of $\geq 1^{\circ}$, but we did not have a sufficient number of saccades of one-half or one-quarter degree in amplitude to be able to determine if these cells might not have shown a burst for these smaller movements. In two cases, we found cells with bursts in a few individual trials for such smaller movements, an observation that also supports the view that there may be a continuum of behavior between buildup and fixation cells. Nevertheless to be able to compare our data in one-dimensional analyses to that of Munoz and Wurtz, we classified all cells for which we could show an acceleration of discharge and a burst (however weak) for saccades of $\geq 1^{\circ}$ as buildup neurons. Those cells that still showed a deceleration and a pause (however weak) for $\geq 1^{\circ}$ saccades were classified as fixation neurons. For the two-dimensional analyses, we treat fixation neurons as the most rostral extension of the buildup neuron layer and do not distinguish between the two types.

Using these discrimination criteria our sample contained 64 buildup neurons, 29 burst neurons, and 9 fixation neurons.

\section{General two-dimensional field properties of deeper layer cells}

Figure 4 shows examples of two-dimensional movement field estimations obtained for two cells with our radial basis function algorithm at three different time frames. The data used to compute the three, movement field frames on the left are from a cell recorded at a depth of $1.4 \mathrm{~mm}$ from the dorsal surface of the colliculus. This cell was classified as a burst neuron by our criteria. At the time of its peak discharge (middle frame), the movement field estimate shows a closed-field shape with a peak discharge for saccades $\sim 7.5^{\circ}$ in amplitude and nearly horizontal in direction. At the other time instants ( $100 \mathrm{~ms}$ before the time of peak discharge in the upper frame and at saccade end in the lower) the cell shows low-level activity located in the same restricted region of movement field space as that at the time of peak discharge.

The movement field profile of this cell could have been fit by a bivariate Gaussian function as well as by our radial basis function method. In fact, when we fit the field of this cell at the time of peak discharge with such a function, the
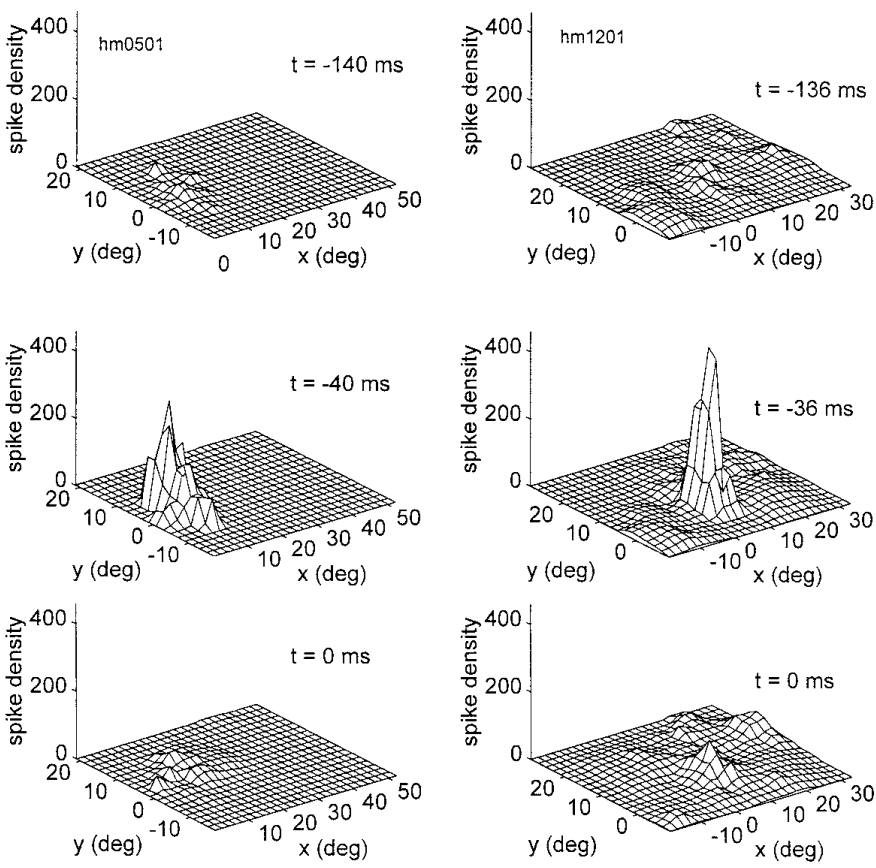

FIG. 4. Example movement fields for a burst neuron (left, cell hm0501) and a buildup neuron (right, cell hm1201) for 3 different time instants obtained with the radial basis function algorithm. All times are given with respect to the end for all saccades. Middle: cell at the time of its peak discharge. Top: $100 \mathrm{~ms}$ before time of peak discharge. Bottom: at saccade end. Movement fields are plotted in horizontal $(x)$ and vertical $(y)$ eye movement coordinates with right and up being positive. Both cells were recorded in the left SC.

surface resembled that shown in middle frame. The summed square error for the surface estimates at the actual data points was generally similar for the radial basis function fits and for the bivariate Gaussian function fits for the 15 burst neurons we tested with both types of fits, although on average, the radial basis function fits produced smaller errors (Anderson et al. 1998). Our preference for use of the radial basis functions fits is illustrated by the estimated movement fields of the buildup neuron (recorded at a depth of $1.6 \mathrm{~mm}$ ) shown in Fig. 4, right. Although this cell has a very circumscribed region of saccade vectors for which it showed a high level of activity at the time of peak discharge (middle frame), it also shows low-level activity for a much wider range of saccades to locations in both the contralateral $(+x)$ and ipsilateral $(-x)$ hemifields at earlier times (upper frame). It shows resumed activity for large contralateral saccades at times close to the end of saccades (lower frame). It is the spatial asymmetries and temporal order of this low-level activity that forms the basis of the Munoz and Wurtz (1995b) theory. Clearly, bivariate Gaussian functions would have difficulty capturing simultaneously the essence of the peak discharge and the low-level activity. Indeed, for this cell, neither Gaussian or a log-Gaussian functions could be made to converge for the cell's data with a standard nonlinear optimization algorithm (Marquard 1963). Because we wanted to use the same estimation technique for all cells, we chose a radial basis function algorithm, which always converged and produced smooth estimates for all cells.

Figure 5 shows another example of a movement field estimate for a buildup neuron (recorded at a depth of 1.8 

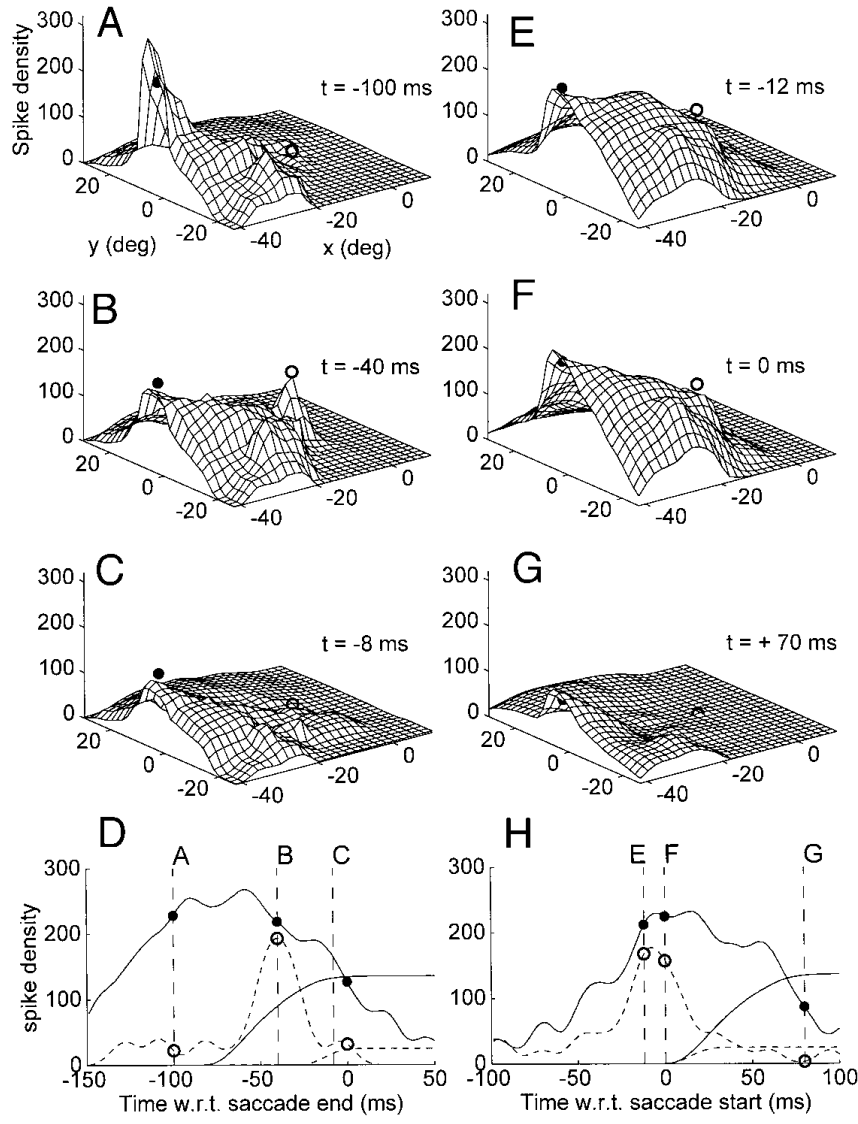

FIG. 5. Example of a buildup cell (ba2201) with extreme open-field characteristics. All 2-dimensional movement-field estimates shown on the left were obtained from data aligned on saccade end. Estimates shown on the right are from data aligned on saccade start. $A$ : time frame $(-100 \mathrm{~ms}$ with respect to end for all saccades ) near time of maximum discharge for the cell. $B$ : at $40 \mathrm{~ms}$ before the end of saccades the cell shows a decline in activity for large saccades but a prominent peak for smaller $\left(\sim 7^{\circ}\right)$ saccades. $C$ : activity for saccades at the time of saccade end. Peak at the $7^{\circ}$ locus has declined rapidly while that for large saccades is still decaying more slowly. Same coordinate conventions as previous figure. This cell was recorded in the right SC. $D$ : temporal characteristics of the cell's discharge. Solid curve shows the average spike density as a function of time with respect to saccade end computed from actual data for the 5 saccades in the database closest to the saccade vector $39^{\circ}$ at $170^{\circ}$. Lower solid curve shows the mean time course of the radial eye position change for these saccades (arbitrary amplitude scale). Dashed curve shows the average spike density for the 5 saccades closest to the saccade vector $7^{\circ}$ at $170^{\circ}$. Lower dashed curve shows the mean time course of the radial eye position for these 5 saccades. The dashed vertical lines show the times represented in $A-C$ above. $\bigcirc$ and $\bullet$, corresponding positions and times for the discharges in $A-D$. $E$ : time frame $12 \mathrm{~ms}$ before saccade start for all saccades. $F$ : at saccade start. $G$ : at a time $70 \mathrm{~ms}$ after saccade start. $H$ : same format as subplot $D$ but now for data aligned on saccade start.

$\mathrm{mm})$. This is the same cell used to illustrate the radial basis function technique in Fig. 1. The shape of the cell's movement field at its outer border could not be determined even for the largest saccade amplitudes of $\sim 40^{\circ}$ that were recorded for this particular cell. Because of the complex spatiotemporal shape of the movement field estimates for this cell, we have aligned its activity on two temporal epochs. Data that were used to generate Fig. 5, left, were all aligned on saccade end. This was our standard alignment and is the alignment required to test the Munoz and Wurtz hypothesis for dynamic population activity, as explained more fully later. The data for Fig. 5, right, were aligned on saccade onset, the more traditional way of examining saccade-related discharge. The figure shows that estimates of the cell's movement field for saccades of $\leq 40^{\circ}$ could be obtained with our algorithm, while Gaussian models were found not to converge. The cell shows significant early discharge for large horizontal saccades. At a later time instant (Fig. 5, $B$ and $F$ ) the peak for $40^{\circ}$ saccades has subsided partially, and a broad region of elevated activity exists. In this same time frame a clear secondary peak of activity (Fig. 5, ○) may be seen for nearly horizontal saccades at smaller amplitudes ( $\sim 7^{\circ}$ in amplitude). The appearance of this peak is particularly clear in the data aligned on saccade end. The temporal time courses of activity aligned on the end of saccades for two selected movement sizes is shown in Fig. $5 D$ and for activity aligned on saccade start in Fig. $5 H$. This plot was computed from temporal averages of actual spike density data, not the radial basis function estimates, for five selected saccades for each curve. The solid curves in each panel show the average for movements closest to the saccade vector $39^{\circ}$ at $170^{\circ}$. The dashed curves show the average for movements closest to the saccade vector $7^{\circ}$ at $170^{\circ}$.

Comparison of the mean of the local discharge for the two sizes of saccades at three time instants (marked by the vertical dashed lines in Fig. 5, bottom) to that computed at the same three time frames and (shown in the spatial plots above) shows that the algorithm provides a good estimation of the spatio-temporal activity even for cells with the complex dynamics recorded in this cell. More rigorous reliability analyses for the radial basis function fits are given in Anderson et al. (1998). Note, in particular, that this cell shows (for data aligned on saccade end) an earlier broad elevation in discharge for the largest saccades and a later peak for small saccades, a trend opposite to that predicted by the Munoz and Wurtz (1995b) theory. We recorded from seven buildup cells with similar complex spatio-temporal patterns in their movement fields.

We noted that the cell shown in Fig. 5 also had a visual response and that the optimal target location (in retinal coordinates) for evoking its visual response was $7^{\circ}$ at $180^{\circ}$. The activity of this cell was recorded on a penetration in which two burst neurons had been recorded dorsal to its location, and their optimal saccade vectors were close to the optimal visual vector for the cell. Taken together all these observations suggest that for some deeper cells, the movement fields can be quite complex with the shape and the location of the center of field changing depending on the time of observation and the point of alignment.

We fit similar two-dimensional estimates as those shown in Figs. 4 and 5 for all 102 cells in our sample. Gross measures of the movement field distributions were used for summary comparisons of the field sizes between burst neurons and buildup neurons. We first found the time frame of maximum activity for each cell. From the two-dimensional movement field estimate for that time frame, we computed the variances of the discharge profile along the field's major and minor axes. The standard deviations of these measurements of field spread were also computed, and these data as a function of eccentricity are given in Table 1. Although these measures of field size ignore asymmetries in field shape and greatly underestimate the actual extent of field size for low- 
TABLE 1. Variances of the discharge profile

\begin{tabular}{lccc}
\hline \hline & \multicolumn{3}{c}{ Radial Distance to Field Center, deg } \\
\cline { 2 - 4 } & $1-10$ & $10-20$ & $20-40$ \\
\hline Burst cells & & & \\
$\quad$ Major axis & $3.34 \pm 1.62$ & $6.13 \pm 2.42$ & $6.81 \pm 3.44$ \\
Minor axis & $2.26 \pm 0.72$ & $4.25 \pm 1.57$ & $4.67 \pm 1.81$ \\
$n$ & 12 & 10 & 7 \\
Buildup cells & & & $12.54 \pm 2.52$ \\
Major axis & $7.97 \pm 3.73$ & $9.19 \pm 2.87$ & $8.83 \pm 2.08$ \\
Minor axis & $4.64 \pm 2.20$ & $6.32 \pm 2.47$ & 17 \\
$n$ & 22 & 25 & \\
\hline
\end{tabular}

Radial distance to field centers was computed as the center of mass of the movement field fit at the time of peak discharge for each cell. Average values of field size (defined as means \pm SD of the discharge distributions along the respective axis) at the time of peak discharge are shown. Both measures are given in degrees.

level activity or for cells with no apparent outer borders (Fig. 5), they do show that field size increases as a function of the radial distance to the center of the field for both types of cells. Overall the field sizes based on this simple measure for buildup cells range from 1.5 to 2.0 times those of burst cells along both major and minor axes and over all three bins of field center eccentricities. The differences in field size were significant ( $t$-tests, $P<0.02$ ) along both axes and over all eccentricities.

\section{Estimating population activity in the $S C$ in space and time}

In this section, we present the results of our computations showing the population activity in the two layers of collicular cells (based on our separation criteria) for saccades of particular sizes and directions. Population estimates are generated from individual movement fields for neurons spatially sampled across the SC. The crucial first step in generating these estimates is to estimate the anatomic location on the collicular surface of each cell in the sample.

DETERMINING THE ANATOMIC LOCATION OF RECORDED CELLS. Past studies of collicular population activity have located cells on the colliculus based on the saccade vector that yields the peak or largest mean saccade-related discharge (Munoz and Wurtz 1995b; Ottes et al. 1986). This method seems justified for burst neurons that have clear and relatively narrow peaks in their movement fields but is more arbitrary for buildup neurons, which often have weak bursts and broadly tuned movement fields in both movement space and time (e.g., the cell shown in Fig. 5). Furthermore, as we mentioned above, the optimal saccade vector for some buildup neurons did not correspond well to that observed in burst neurons or to the visual responses recorded in other cells on the same penetration. Therefore, we wondered if the use of the center of a cell's visual field response to determine their location on the colliculus might not provide a more consistent method of locating cells than methods based on measurements on their movement field.

Visual responses. We examined stimulus-locked activity of each cell in the delayed saccade paradigm. After alignment on target onset, the activity of almost all the burst and buildup cells ( 88 of the 92 studied in this report) showed an abrupt increase in discharge within 50-80 ms for a spatially limited region of target positions. From a typical data set we selected the eight individual trials with the largest mean discharge over this interval of time and computed the geometric center (in horizontal and vertical coordinates ) of their target vectors. Because the visual fields tended to have sharp spatial peaks, this measurement is essentially equivalent to measurement of the peak of the visual field. Four cells (all of which were buildup neurons) lacked a visual response. These cells were placed at the same location as the nearest cell, recorded on the same microelectrode penetration, which did have a visual response. For these four cells, the greatest separation between the cell without a visual response and the locating cell with a visual response was $0.4 \mathrm{~mm}$ (a distance mostly in depth, not position on the collicular map because our tracks were run roughly perpendicular to its surface).

Motor responses. Two options were considered for the optimal saccade encoded by a cell based on the motor response. The first measure used to locate cells on the map was the saccade vector producing the largest peak discharge on any trial in the raw database. This measure also was used by Munoz and Wurtz (1995b) to locate their cells. The second measure used the eye movement vector associated with peak activity from the radial basis function surface fit. For each neuron, the optimal vectors determined from the three measurements then were converted into three sets of candidate locations on the SC from the formulae provided by Ottes et al. (1986).

Electrically evoked responses. Electrical stimulation also was delivered in the deeper layers of the SC as the monkey fixated (see METHODS). The evoked fixed vector saccade from such stimulation also was converted into an anatomic location on the SC. Because the collicular motor map is based on graphic analysis of the saccade vectors evoked by electrical stimulation (Ottes et al. 1986), it is logical to assume that stimulation provides the most accurate estimate of the location of the microelectrode. However, stimulation data were not available for all cells. Hence, a comparison of the differences in the placement of the cells using the three methods described earlier, with respect to the stimulation site, was done for 15 buildup neurons. Our results showed that the visual field measurements provided the closest correspondence to the locations determined by stimulation. The mean difference between locations determined by the visual field method and the that from electrical stimulation was $0.51 \mathrm{~mm}$ (range $0.19-1.09$ ). The mean difference for the locations based on the peaks of the movement field from the radial basis function fits and from the raw data were $1.02 \mathrm{~mm}$ (range $0.09-2.52$ ) and $0.76 \mathrm{~mm}$ (range $0.26-$ $2.09)$, respectively. The differences between the visual field method and either method of movement field measurement were both significant ( 1 -tailed $t$-tests; $P<0.01$ ). The differences between the two movement field methods were not significant ( $t$-test; $P>0.3$ ). The same analysis was repeated for seven burst neurons. None of the differences in method of cell location were significantly different. The results of these analyses confirm our suggestion that for some buildup neurons larger errors can result from placing the cell based on movement field measurements than on visual field measurements.

There remains a concern that the use of visual fields to 

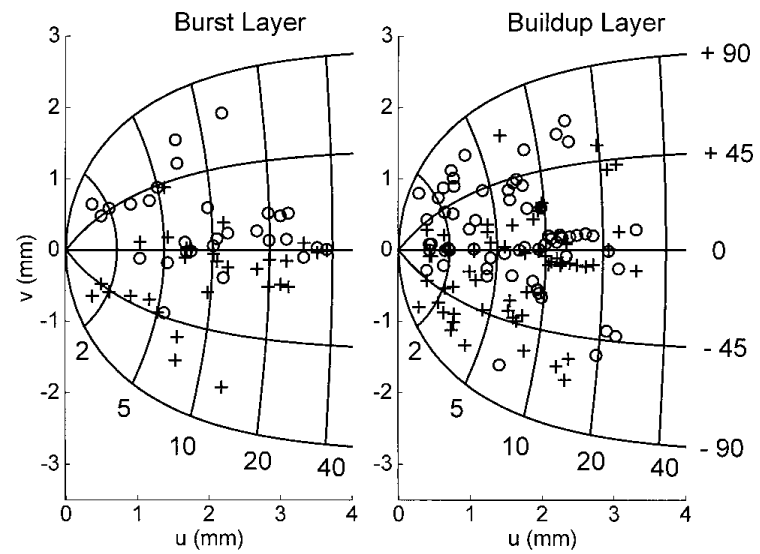

FIG. 6. Locations of the recorded cells $(O)$ and reflected cells $(+)$ plotted on the collicular motor map. To locate the cells, horizontal and vertical coordinates of the peak of each cell's visual field were converted to collicular coordinates using the formulae of Ottes et al. (1986). See text for details. Left: estimated anatomic location on the collicular map for burst cells. Right: estimated anatomic location for buildup cells. In each, the abscissa (u) shows the rostral (0) to caudal (4) axis of a colliculus in mm. The ordinate $(v)$ shows the medial $(3)$ to lateral $(-3)$ axis also in millimeters. Curvilinear coordinate system plotted on the collicular surface shows location in polar coordinates of the visual field (eccentricity marked in degrees along the bottom edge and direction in degrees along the caudal edge). Positive numbers signify up directions.

locate cells may introduce a systematic bias in cell placement in the rostral direction that could compress the whole population map (McIlwain 1976) toward the rostral pole. To test this notion, we computed the rostrocaudal component of the difference between the placements determined by the visual fields and those determined by the movement field peaks (from the raw data) for the same 15 buildup cells considered earlier. In this computation, a negative sign indicates that placement derived from the use of the visual field was more rostral than that derived from the use of a movement field metric. For the 15 cells the results were negative in 7 and positive in 7, and there was no rostrocaudal shift for 1 cell. Thus the use of a visual field metric did not introduce a systematic bias in the location of cells on the map. Therefore we adopted the latter method in the present analysis.

Figure 6 shows the locations of the cells obtained with the method of visual fields. Figure 6, left, shows the locations over the collicular motor map for burst neurons, and the locations of buildup neurons are mapped in Fig. 6, right. This figure represents one colliculus with its most rostral limit located at zero on the abscissa and its most caudal extent at $\sim 4.6 \mathrm{~mm}$. Its medial edge is shown as up (positive on the ordinate) and its lateral edge as down (negative on the ordinate). The cells, which actually were recorded in both colliculi, were transposed to the one model SC depicted in Fig. 6 and were plotted as open circles. Finally, all cells were reflected across the horizontal meridian ( $v=0$ axis $)$, and the reflexed cells are represented by crosses. For each such duplication, the cell's movement field data also were reflected about the vertical meridian for that neuron. Transposition and duplication allow a more uniform coverage of the $3.5 \mathrm{~mm}$ rostral extent of the colliculus but make the assumption that cells at the same eccentricity in the left/ right and medial/lateral colliculus behave in mirror image fashion for left/right or up/down oblique saccades, respec- tively. This sampling also produced more reliable population activity surfaces using our radial basis function algorithm. The maps in Fig. 6 show that we obtained fairly uniform spatial coverage, particularly for cells in the buildup layer, for eccentricities out to $25^{\circ}$ and oblique angles of $\pm 45^{\circ}$. Burst cells are less well covered at oblique angles for eccentricities of $>15^{\circ}$. Therefore, in the following analyses we limit determination of population activity to $15^{\circ}$ eccentricity for oblique angles for the burst cell layer but will include analyses out to $25^{\circ}$ at all elevations $\leq 45^{\circ}$ for the buildup layer.

ONE-DIMENSIONAL POPULATION ANALYSIS. Although the primary goal of the present investigation was to produce twodimensional motor maps of the colliculus, we first present the results of a one-dimensional analysis for horizontal saccades so that a direct comparison can be made to the analysis of Munoz and Wurtz (1995b). Next, we perform two statistical tests to determine whether the population activity in the buildup layer spreads to rostral SC sites in a systematic manner or rather that rostral neurons are reactivated randomly.

The subset of neurons located within $\pm 45^{\circ}$ oblique contour curves of the SC were used for the one-dimensional analysis. The selection procedure limited our sample to 25 burst, 42 buildup, and 9 fixation neurons. The location and corresponding movement fields were rotated such that optimal saccade amplitude remained the same but the preferred direction was now zero. The population activity analysis then was performed for a $20^{\circ}$ saccade. The visualization of the rostrally directed spread in activity in buildup cells was most convincing in the results presented by Munoz and Wurtz (1995b) for saccades of $50^{\circ}$ amplitude, but our population data are limited to examining movements only $\leq 20-$ $25^{\circ}$ in amplitude as already noted.

Buildup neuron discharge was normalized to the peak activity associated with the largest saccades in the cell's preferred direction. Burst cell discharge was normalized to the peak activity obtained for the optimal saccade vector. Fixation neuron discharge was normalized to the maximal discharge rate during active fixation before saccade onset. This was the same normalization procedure used by Munoz and Wurtz. The nine fixation neurons were placed at random positions within the rostral $0.5 \mathrm{~mm}$ of the $\mathrm{SC}$ (out to $1^{\circ}$ in visual space).

The results of the one-dimensional analysis for a $20^{\circ}$ horizontal saccade are shown in Fig. 7. Figure 7, left, shows the distribution of population activity in the burst layer, whereas Fig. 7, right, shows the same in the buildup layer. Fixation neurons are hypothesized to form a rostral extension of the buildup layer (Munoz and Wurtz 1993), so they are included in the panels on the right with the latter cells. The smoothed population estimates for burst and buildup neurons (continuous trace in each subplot) were obtained by a cubic spline fits. The short dashed lines show the mean value of the discharge data for fixation neurons.

Data in Fig. 7 illustrate population activity for five different time epochs around the movement. The same qualitative pattern of spatio-temporal activity described by Munoz and Wurtz is seen in the data presented here. For burst neurons, the most intense locus of activity is centered at $\sim 3.1 \mathrm{~mm}$ 

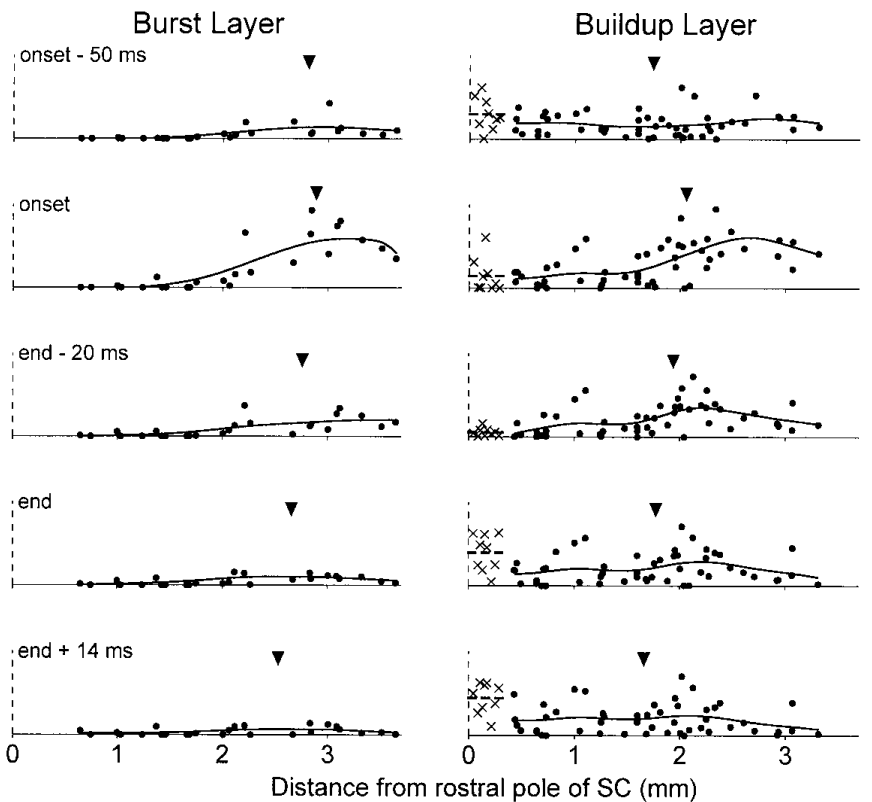

FIG. 7. One-dimensional spatial distribution of activity along the horizontal meridian of one superior colliculus for a $20^{\circ}$, contralateral, horizontal saccade. Left: activity in the burst neuron layer ( $n=25$ cells). Right: activity in the buildup layer ( $n=51$ cells) and for fixation cells $(n=9)$. -, normalized activity of 1 burst or buildup neuron at 5 different time frames around the time of the saccade. $\times$, normalized activity of 1 fixation neuron. See text for methods of normalization. For burst and buildup neurons, the location of each cell along the horizontal meridian of the SC (the abscissa) was determined from the amplitude of its optimal visual response converted to millimeters from the rostral limit of the SC as explained in the text. Fixation neurons were placed at random within the narrow region from the rostral limit to $0.5 \mathrm{~mm}$ more caudal. —, spline fits for each group of cells (for burst and buildup cells ). - - - , mean level of activity in the fixation cells. $\boldsymbol{\nabla}$, computed position of the center of mass of the distributed activity in each time frame.

on the SC before and at saccade onset. By saccade end, discharge is essentially over at all locations. There is little or no activity in rostrally located burst neurons at any point in time during the $20^{\circ}$ saccade. In contrast, almost all buildup neurons (even those located most rostrally from the site coding the impending $20^{\circ}$ saccade) show activity in the time frame at $50 \mathrm{~ms}$ before saccade onset, but it is difficult to determine the location of a peak in this activity at this time. Note that by $50 \mathrm{~ms}$ before saccade onset the "buildup" of activity for both types for cells in the zone just caudal to $\sim 2.0 \mathrm{~mm}$ is similar in magnitude, underscoring our belief that the presaccadic activity for saccades near the optimal vector may not be a good discriminate between the two classes. However, the cells that were located rostral to the $2.0 \mathrm{~mm}$ marker were clearly more active if they were in our buildup class (Fig. 7, right). Perhaps a better discriminating criterion, at least for rostral cells, would be to examine their early activity for the largest movements in the cell's data set.

At saccade onset, a clear peak of activity (as shown by the spline fit to the data) emerges at a position somewhat rostral to the location of peak activity seen on the burst neuron curve while some rostral buildup and almost all fixation cells tend to decrease their activity. As the saccade proceeds the rostral activity in buildup neurons begins to return to its presaccadic level, and the locus of the most intense activity (as represented by the spline curve) undergoes a slight, rostrally directed shift in location. After saccade end, rostral and intermediate activity returns to same level as that which it showed before saccade onset, but activity in the caudal region just beyond the region associated with the most intense activity at saccade onset has largely collapsed to levels below presaccadic discharge. All of the trends just described for population activity in the buildup layer are small and seem very dependent on a few cells and the impression created by the spline curve fits.

The nine most rostral neurons show the same pattern of activity described for fixation neurons by Munoz and Wurtz (1995b). Note that the apparent difference in the magnitude of the activity in these most rostral cells and rostral buildup neurons is probably due to the different method of normalization used on the activity in these two groups of cells. More attention should be paid to the similar temporal dynamics in the discharge of two groups of cells than in their normalized amplitudes.

The arrows show the computed center of mass of the population activity for burst and buildup neurons. For both types of cells, there is a shift in the center of mass from the time of peak activity ( saccade onset) to saccade end, but the shift is only $\sim 0.25 \mathrm{~mm}$ in both layers. Computation of this shift for the buildup layer does not include the contribution of fixation neurons.

Munoz and Wurtz interpreted the spatio-temporal pattern of activity seen in buildup neurons as evidence for a systematic, rostrally directed spread of activity in this group of cells that occurred during the saccade and further hypothesized that this could be used to control saccade dynamics. Because the activity in buildup cells located at the point of largest discharge at saccade onset collapses more rapidly than that in cells displaced slightly more rostrally, there is a rostrally directed shift in the position of the center of mass activity in the buildup layer during saccades. However, it is not clear from our data or theirs whether a wave of activity passes through the buildup neurons located more rostral to the location of the peak of activity at saccade and whether this wave then reactivates fixation neurons as required by their theory of saccade control.

Although one cannot attach goodness-of-fit measures to spline interpolations, certain statistical tests can be used on the raw data to test the systematic spread of activity hypothesis, which requires the sequence of activation of neurons to follow a caudal to rostral order. We used the runs test (Hoel 1965; Walpole and Myers 1989), a nonparametric significance measure that looks for proof of sequential changes in the dependent variable (reactivation time, see further) measured over a range of the independent variable (caudal to rostral location on the $\mathrm{SC}$ ), to test for sequential activation during $20^{\circ}$ saccades. Examination of Fig. 7 (right) shows that all buildup neurons caudal to $\sim 1.7 \mathrm{~mm}$ were maximally active by onset of $20^{\circ}$ saccades. Thus a spread of activity, if it occurs, must involve all buildup and fixation neurons (total of 34 in our sample) rostral of this location. The time of reactivation, defined as the period of time from $25 \mathrm{~ms}$ before saccade onset to $14 \mathrm{~ms}$ after saccade end when a cell reached one-half of the maximum level it attained over the same time range, was computed and aligned with respect to saccade end. The one-half level of maximal activation, not 
the peak activity during the saccade, was used because many neurons had not reached their maximal level even by $14 \mathrm{~ms}$ after saccade end, although they did return to presaccadic activation levels. The null hypothesis of the runs test is that the reactivation times, sorted according to cell location from caudal to rostral, are not different from a random sequence. We could not reject this hypothesis with our data $(P>0.1)$, suggesting that the reactivation of neurons in the rostral buildup layer may be random instead of sequential as required by a strict interpretation of the Munoz and Wurtz (1995b) theory.

In a separate statistical test on the data shown in Fig. 7 (right column for buildup neurons), we used a bootstrap technique (Efron 1982; Kooperberg et al. 1995) to compute the $95 \%$ confidence levels for the spline fits. One-half of the neurons located rostral to the $1.7-\mathrm{mm}$ limit were selected at random from the original data $(n=34)$. The data and the locations for the reduced data set were used to recompute the spline curves at each of the five time epochs. This procedure was carried out repetitively for 200 cycles. The five highest and lowest values of the ensemble of spline fits at each spatial location were discarded, and the resulting range of ensemble values are plotted as the confidence limits in Fig. 8. The original spline curves ( - ) are plotted for the full data set from Fig. 7. Next the locations of all the neurons located rostral to $1.7 \mathrm{~mm}$ were shuffled randomly, and the data for the shuffled locations were fit with splines (- - -) for each time epoch. The distribution of activity at all times and for all rostral collicular locations as estimated by the spline fits to the shuffled data remains inside the $95 \%$ confidence levels, generating further doubt about the notion that there is a serial-order rostral spread of activity on the SC during saccades. These results would be compatible with a hypothesis that many buildup neurons located some distance rostral to the region of maximal activity during saccades are suppressed or totally silenced just before saccade onset and then become reactivated as a group with temporal jitter but not in serial order based on location as the saccade progresses. The location of the region of rostrally inhibited neurons appears to vary with saccade size.

TWO-DIMENSIONAL ESTIMATES OF COLLICULAR POPULATION ACTIVITY. We now present data showing the two-dimensional distribution of activity in the two layers of the colliculus during saccades as estimated by our radial basis function algorithm. In the following estimates of and computations on two-dimensional activity, we included all cells, even those cells previously classified as fixation neurons, in the analysis precisely because we could not determine any statistical difference in the pattern of discharge of rostral "buildup" neurons and "fixation" neurons. We did not normalize the activity of any of the cell groups before computing the distributed activity maps. Figure 9 shows the population activity in the contralateral colliculus for a $20^{\circ}$ horizontal saccade. Figure 9, top, shows the distribution of activity in the burst cell layer at three different time frames (100 ms before saccade onset, saccade onset, and saccade end). Figure 9, bottom, shows the activity in the buildup layer at the same three times.

Activity in the burst layer is circumscribed in space at all three times with the center of mass located at $2.9 \mathrm{~mm}$ at
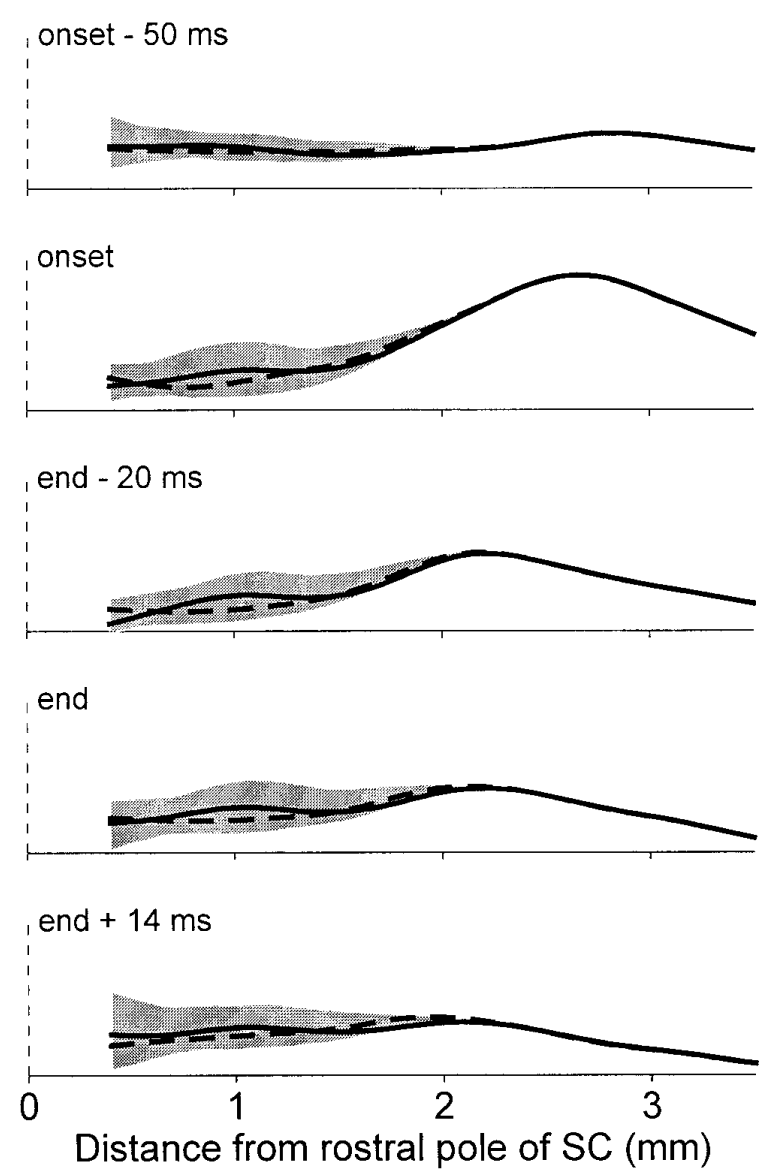

FIG. 8. Confidence limits on the spline fit estimations of 1-dimensional distribution of buildup layer activity for a $20^{\circ}$ contralateral horizontal saccade. Same time frames shown in Fig. 7 are included from top to bottom. - , same spline fits to the actual data shown in Fig. 7 (right). Shaded regions show the $95 \%$ confidence limits for the spline fits generated by a bootstrap technique described in the text. - - -, spline fits to distributed activity when the locations of the cells within the rostral $1.7 \mathrm{~mm}$ of the SC first had been shuffled randomly. Note that the fits for the actual data and the spatially shuffled data both remain inside the $95 \%$ confidence boundaries for all time frames, suggesting that either fit is an equally good statistical representation of the distribution of activity along the horizontal meridian of the SC for all time instants around the time of this saccade.

saccade onset. The activity surface is roughly symmetrical about this point along the horizontal meridian of the colliculus. Activity extends $\sim 1 \mathrm{~mm}$ both rostrally and caudally and slightly more in the mediolateral dimensions. Only low-level activity is evident $100 \mathrm{~ms}$ before saccade onset (Fig. 9, left), but it is located at nearly the same spatial locus as at saccade onset, which was close to the time of peak discharge. Activity declined rapidly during the saccade so that only scattered low-level discharge remained at saccade end as shown in the section on the right. Computations of the center of mass of the activity (discussed more fully in Fig. 13) showed no shift during the movement.

Activity in the buildup layer was more widespread at all times in comparison with the burst layer and was distributed asymmetrically along the horizontal meridian of the colliculus. There was a low-level region of activity located in the rostral colliculus well before saccade onset (Fig. 9, bottom left, subplot at $100 \mathrm{~ms}$ before saccade onset) produced by 

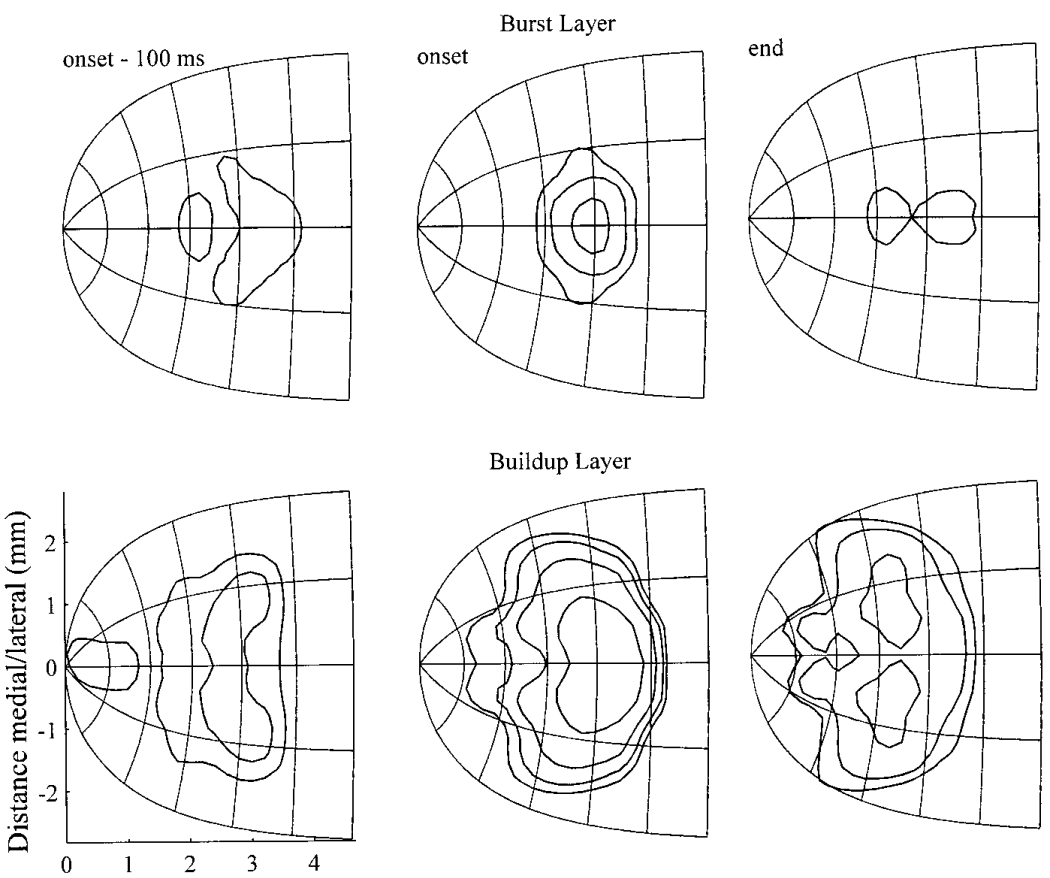

FIG. 9. Two-dimensional population activity in 2 layers of the SC for a $20^{\circ}$ horizontal, contralateral saccade. Each section shows the distributed activity on the collicular motor map. Top: activity shown as constant contour curves in the burst neuron layer. Bottom: same representation for the buildup neuron layer. Left: $\sim 100 \mathrm{~ms}$ before saccade start. Middle: distribution of activity at saccade start. Right: saccade end. Contour curves are 15, 30, 60, and 120 spikes/s. rostrally located neurons. Note that the lowest contour line (15 spikes/s here and in all other subplots for purposes of comparison) extends to the most rostral limit of the SC and the same contour also circumscribes the more caudal island of low-level activity. There is a slight valley between the two regions that would disappear if a lower limit had been chosen as the lowest contour curve. The center of mass of the activity surface is located at $2.8 \mathrm{~mm}$ along the horizontal meridian at this early time. By saccade onset, the location of the rostral limit of activity has receded caudally and the contours of equal activity have become concave inward toward the more caudally located peak of activity. During this same time span, activity has increased dramatically at the center (just as in the burst layer), and the limit of low-level activity has been extended along the caudal and mediolateral axes. By saccade end, the activity has declined steeply at the former center location, producing a shift in the center of mass of the activity in the rostral direction accompanied by further spread of low-level activity in the medial and lateral directions until they occupy the whole width of the colliculus. Activity in the caudal end retracts to about the same limit observed in the presaccadic interval, but the rostral limit of the activity has shown little change.

Figure 10 shows similar estimates of the distribution of activity in the colliculus for a $2.5^{\circ}$ horizontal saccade. The distribution of activity in the burst cell layer is circumscribed and symmetrically distributed along the horizontal meridian, as it was for the $20^{\circ}$ saccade. The activity in the buildup layer was also more widespread in comparison with the burst layer and lacked the marked asymmetrical distribution present on the horizontal meridian that occurred in the buildup layer for $20^{\circ}$ saccades. The rostral region was again inactive by the onset of the $2.5^{\circ}$ saccade and was partially reactivated by its end. The width (in millimeters on the colliculus) of activated cells in the mediolateral direction was somewhat greater at saccade onset than for $20^{\circ}$ saccades.
Figure 11 shows the distribution of activity in the two layers for a $15^{\circ}$ saccade made at an oblique angle of $45^{\circ}$ up. The burst cell layer discharge was similar to that illustrated for 20 and $2.5^{\circ}$ horizontal saccades. The activity distribution in the buildup layer was again more widespread in comparison to the burst layer. Also, there was a small, but clearly evident, tilt of the axis of rostrally directed asymmetry of activity in both layers that appear to be aligned with the $45^{\circ}$ up meridian in collicular space.

ROSTROCAUDAL AND MEDIOLATERAL SECTIONS THROUGH THE ACTIVITY SURFACES. To show the spatial details of the population activities in the colliculus, we took sections thorough the surface estimation shown in Fig. 9 for a $20^{\circ}$ horizontal saccade at movement start and end. Figure $12 \mathrm{~A}$ shows a section made along the horizontal meridian from rostral to caudal with the bust layer activity on top and the buildup layer activity below. The asymmetric distribution of activity in the buildup layer, which is broadly skewed toward the rostral colliculus, is seen clearly (Fig. 12A, bottom section). The rapid collapse of caudal activity in the buildup layer, which shifts the center of mass of the activity in this layer toward the rostral limit of the SC by saccade end, is also noticeable. However, it is difficult to detect any change in the activity profile in the region located more rostral than $\sim 1.7 \mathrm{~mm}$. Therefore the primary dynamic event in the buildup layer, as it also is in the burst layer, is the decline of activity at the locus of peak discharge at onset.

Figure $12 \mathrm{~B}$ shows a section made orthogonal to that of Fig. $12 A$ (mediolateral across the collicular surface at 2.9 $\mathrm{mm})$. Burst layer activity is shown in Fig. 12B, top, and buildup layer activity in Fig. $12 \mathrm{~B}$, bottom. The distribution of activity is again shown at saccade onset and end in each layer. Activity is symmetrically distributed in each layer, but the extent (distance) is more widespread in the buildup layer. The activity declines "in place" during the saccade in both layers. 

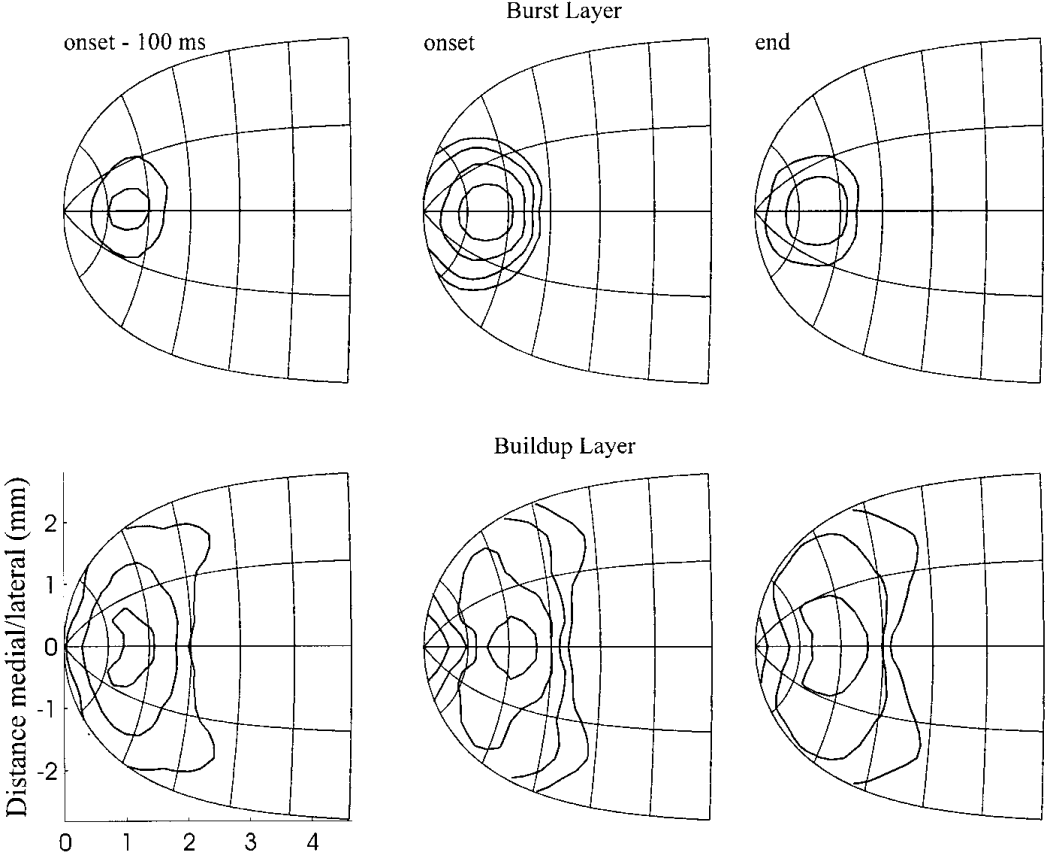

FIG. 10. Two-dimensional population activity in 2 layers of the SC for a $2.5^{\circ}$ horizontal saccade. Same conventions as Fig. 9.
QUANTITATIVE MEASUREMENTS ON THE ACTIVITY SURFACES FOR DIFFERENT SACCADE VECTORS. Figure 13 shows the computed vector movements of the center of mass of activity in the two collicular layers for horizontal saccades with amplitudes from 2.5 to $25^{\circ}$ (lower set of arrows in each section) and for $45^{\circ}$ up oblique saccades over the same range of amplitudes (upper set of arrows), except for the burst layer (Fig. 13, top), where computations were made over the amplitude range from 2.5 to $15^{\circ}$. Each arrow plotted on the collicular surface represents the magnitude and direction of the movement of the center of mass of the distributed activity in that layer during a saccade. The tail of each arrow is placed at the center of mass at saccade onset and the tip of each is at the center at saccade end. There is little or no systematic movement of the center in the burst layer for any of the illustrated saccades. In the buildup layer, the largest horizontal saccades $\left(25,20\right.$, and $\left.15^{\circ}\right)$ are associated with a rostrally directed movement of the center, but the shift was only $\sim 0.25 \mathrm{~mm}$ in distance and was the same size for the three movement amplitudes. Larger oblique saccades also are accompanied by shift in the center of mass of activity of similar distance, and the movement vector is tilted to
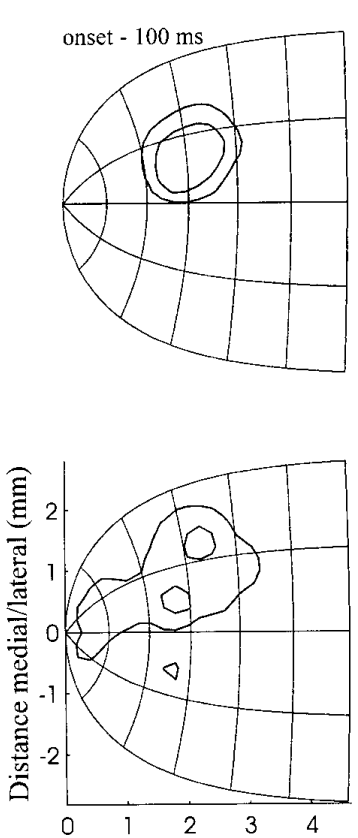

Distance rostral/caudal $(\mathrm{mm})$
Burst Layer
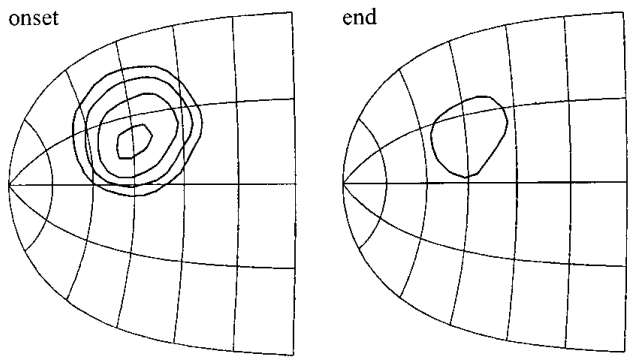

Buildup Layer
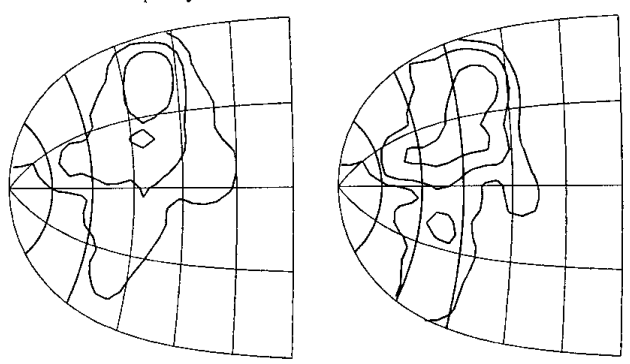

FIG. 11. Two-dimensional population activity in 2 layers of the SC for a $15^{\circ}$ up-oblique saccade. Same conventions as Fig. 9. 


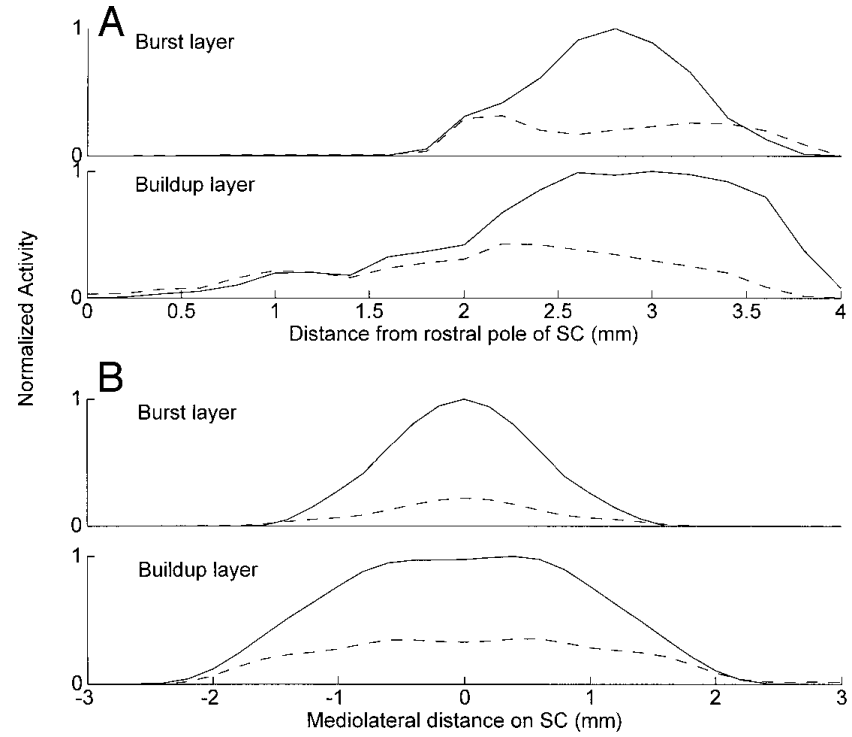

FIG. 12. Cross-sections through the 2-dimensional population activity surfaces for $20^{\circ}$ saccade (Fig. 9) at saccade onset and end. $A$ : section along the horizontal meridian of the SC for the burst layer (top) and the buildup layer (bottom). $B$ : orthogonal section from that shown in $A$ along a mediolateral anatomic axis of the SC at $2.8 \mathrm{~mm}$ from its rostral limit. This section passes very close to or through the peak of the population activity at saccade onset in both layers. In all panels, the population activity first was normalized to the peak of that layer's activity for all locations and times before the sections were made. - , activity at saccade onset; - - - activity at saccade end.

be aligned roughly with the $+45^{\circ}$ oblique meridian on the colliculus.

We next quantified the size of the active area and the amplitude of the peak activity present in the two layers as a function of saccade size for horizontal and oblique saccades. The size (expressed as a proportion of the colliculus) of the active region was defined as that area inside the 15 spike/s contour curves on population activity plots similar to those shown in Figs. 9-11. The measurements of active area and peak activity always were made at saccade onset and are shown in Fig. 14. The results show that the size of the active area in both layers remains relatively constant for all saccades but that the active area is about twice as large in the buildup layer (Fig. 14A). In contrast, there tends to be a consistent decrease in the peak amplitude of activity at saccade onset as a function of saccade size in both layers at least for movements $>10^{\circ}$.

To develop a measure of the total activity in each layer, each small increment of active area $(0.2 \times 0.2 \mathrm{~mm})$ for each layer is weighted by that increment's discharge, then summed over all the active increments at each instant of time during a saccade. In Fig. 15A, we plot the results obtained from this computation for the summed burst layer signal ( - - ) and for the summed buildup layer signal ( $(-)$ for a $2.5^{\circ}$ horizontal saccade. In this figure, time is marked on the abscissa with respect to the end of the saccade (at time 0 ). The dashed vertical lines show the approximate time of saccade onset (left) and saccade end (right). In Fig. $15 B$, similar summed temporal signals for the two layers are plotted for a $20^{\circ}$ horizontal saccade.

For the smaller movement (Fig. 15A), the summed dis- charge well before saccade onset is gradually increasing in the buildup layer, whereas that in the burst layer remains constant until a point closer in time to saccade onset. This difference in presaccadic discharge between the summed activities for the two layers is not as apparent in the signals for the larger $20^{\circ}$ saccade (Fig. $15 B$ ). For both sizes of saccade, the rate of decline of activity beginning after the onset of the saccade was more noticeable in the burst cell layer and total activity was lower in burst cells by saccade end for all sizes of movements.

As a final measure of the population activity, we computed the cumulative activity integrated over time during saccades of different sizes. Figure 16 shows the results of these computations made for horizontal saccades from 2.5 to $25^{\circ}$ in amplitude. Because the duration of saccades increases linearly with size, it might be expected that this energy-like measure of each layer's activity would increase with saccade size. For the buildup layer, this expectation was found to be true, and cumulative activity increased almost linearly with saccade size as does saccade duration. For the burst layer, a counter trend already discussed (decreasing peak discharge as a function of saccade size) kept cumulative activity almost constant as a function of saccade size. Peak activity levels in the distributed discharge at each time frame during saccades declined in this layer as saccade size increased (Fig. 14B). Together the countervariations in discharge level and saccade duration as a function of saccade size produce a constant cumulative discharge in the burst layer.
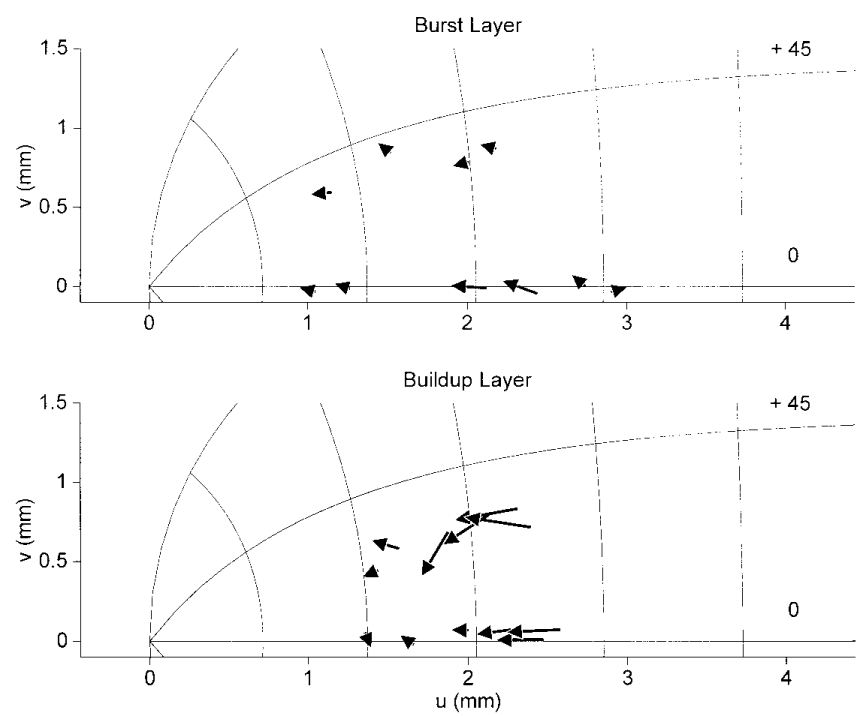

FIG. 13. Saccade-related movement of the center of mass of the population activity in the burst (top) and buildup layer (bottom) layers of SC. Each section represents the medial half of the collicular motor map (Fig. 6 ) with the rostral limit of the colliculus at the 0 point on each abscissa and medial distance from the midcollicular axis on each ordinate. Each arrow is arranged so that its tail represents the center of mass of the population activity at saccade onset and its tip represents the same measure at saccade end. Arrows in each section plotted along the midcollicular axis are for (starting most caudally) $25,20,15,10,5$, and $2.5^{\circ}$ contralateral, horizontal saccades. Arrows located more vertically in each section represent $45^{\circ}$ up-oblique saccades over the same range of movements in the buildup layer but a smaller range of amplitudes $\left(15,10,5\right.$, and $\left.2.5^{\circ}\right)$ in the burst layer. Where the arrows overlapped they have been shifted vertically by a small amount for clarity. 


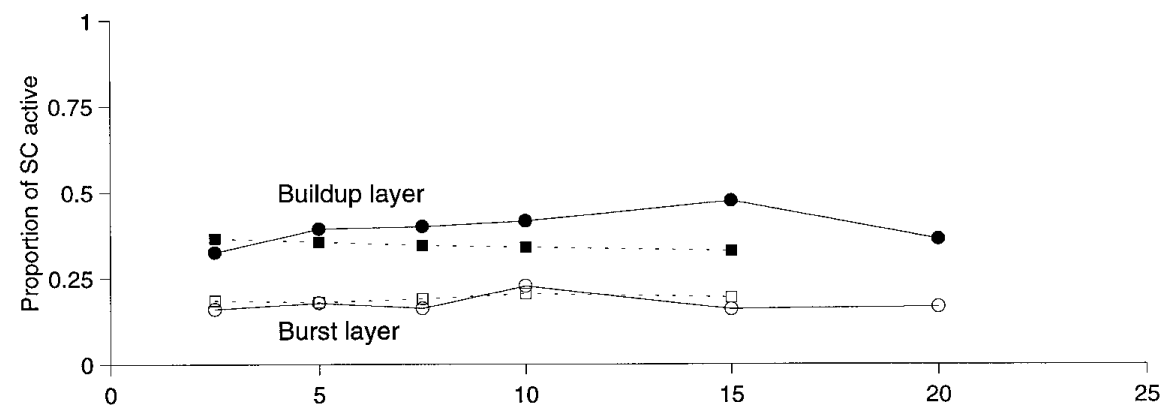

B

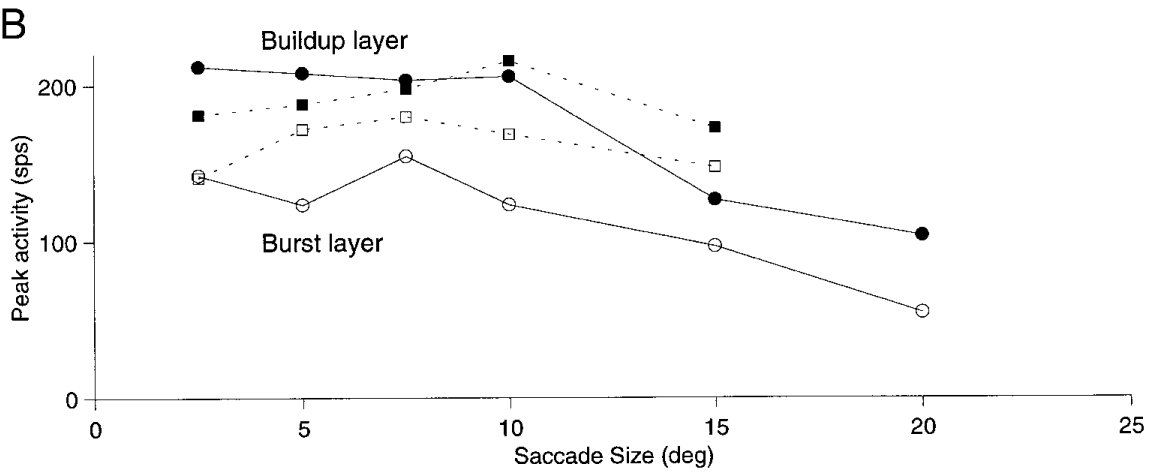

FIG. 14. $A$ : proportions of the total surface area of the contralateral colliculus active (defined as discharge $>15$ spikes/s ) at saccade onset for saccades of different sizes and directions. Active areas computed from population surfaces ( similar to examples shown in Figs. 9-11). B: peak activity measurements at saccade onset for saccades of different sizes and directions. Each data point was computed from population activity surfaces. $\bullet$ and $\mathbf{\square}$, measurements for the buildup layer; $\bigcirc$ and $\square$, corresponding measurements in the burst layer. horizontal saccades; - -,$- 45^{\circ}$ oblique saccades.

\section{ISCUSSION}

\section{"Moving wave"' hypothesis}

The major purpose of our two-dimensional analysis of population activity in the superior colliculus was to test the hypothesis that a rostral spread of activity in the buildup cell layer occurs during saccadic eye movements and is used to control the end of these movements (Munoz and Wurtz $1995 b)$. We first presented the results of a one-dimensional analysis for horizontal saccades. The one-dimensional analysis was conducted on a subset of our total sample of collicular neurons - those located close to the horizontal meridian of the SC. We conducted this analysis in a similar fashion to the one-dimensional analysis carried out by Munoz and Wurtz to show that we had sampled a analogous group of collicular neurons.

The results of the one-dimensional analysis for a $20^{\circ}$ horizontal saccade compare closely with those presented by $\mathrm{Mu}-$ noz and Wurtz (1995b). The visual impression produced by the changes in the spline fits to the population activity as time progresses from before saccade onset, through the movement, and on to its end is compatible with their hypothesis that a spread of activity occurs from a location near the center of mass of activity around the time of saccade onset to the most rostral buildup cells by saccade end or shortly thereafter. Quantitative measurements on the magnitude of the shift in the center of mass of activity in the buildup layer $(\sim 0.25 \mathrm{~mm})$ agree for $20^{\circ}$ horizontal movements where our study overlaps with theirs. However, the order of activation in those buildup neurons located rostral to the location of the center of mass $\left(\sim 1.7 \mathrm{~mm}\right.$ for $20^{\circ}$ saccades $)$ during the saccade cannot be determined in a statistical manner from visual inspection of the spline curves even on our 2-ms time resolution plots of the one-dimensional data. Statistical analyses of the distributed activity in buildup cells rostral to the $1.7 \mathrm{~mm}$ location could not reject the null hypothesis that activation of these cells was in random order as opposed to a serially ordered sequence of activation from caudal to rostral as would be expected if an organized spread of activity through the region were occurring. We believe that the data from both studies are equally compatible with the hypothesis that the tonic presaccadic activity of both fixation cells and rostrally located buildup neurons is inhibited before the onset of large saccades as activity is increasing in more caudally located buildup cells at the optimal point on the map for that particular saccade. As the saccade progresses and its end approaches, activity at the latter location collapses rapidly, while the whole rostral region of inhibited cells (both buildup neurons and fixation neurons ) becomes reactivated over a short period of time but essentially in random order.

The results of our two-dimensional analysis (Figs. 9-11) raise further doubts that a rostrally directed spread of activity occurs in the SC during saccades and, further, that the small shifts in activity that we did observe could be used to terminate saccades. In the buildup layer during $20^{\circ}$ horizontal saccades, the spread of activity along the mediolateral axis into both the upper and lower fields is greater than in the rostral direction. This observation was also quantitatively true for $10^{\circ}$ movements but was not illustrated here. There was also a large mediolateral spread of activity during oblique saccades instead of along the collicular meridian representing the direction of movement that is the direction of spread predicted by the Munoz and Wurtz hypothesis (Optican 1995).

However, it is also clear that rostrocaudal asymmetries in the extent of low-level activity do exist in the buildup layer for large saccades (Fig. 12). In presaccadic time frames, activity exists all the way from the center of mass to the 
A

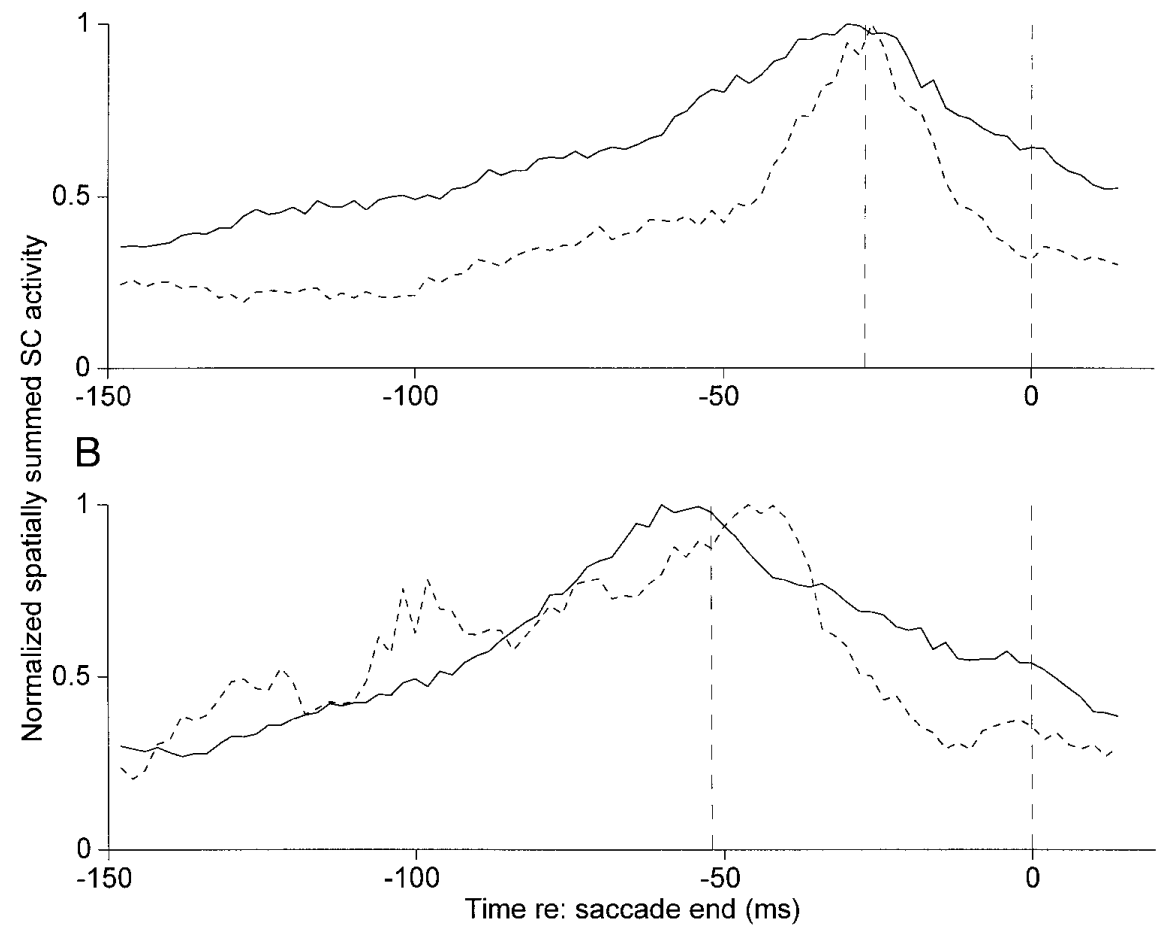

FIG. 15. Summed population activity in 2 layers of the SC as a function of time with respect to saccade end. $A$ : summed activity for a $2.5^{\circ}$ horizontal saccade. $B$ : summed activity for a $20^{\circ}$ horizontal saccade. - - buildup layer activity; - - - , burst layer activity. Dashed vertical lines indicate the times of saccade onset and end. Each curve was normalized so that its maximum equaled unity. rostral end of the SC, whereas the caudal extent of the activity from the center of mass is much more restricted. But, by saccade end little spread of the rostral extent has occurred and asymmetry has not increased. Both anatomic (BüttnerEnnever and Horn 1994) and electrophysiological (Gandhi and Keller 1997) studies have shown that a negative gradient of projection strength exists from the rostral to caudal SC to the region of the omnipause neurons. It seems likely that these inputs from the SC serve as one source of excitatory

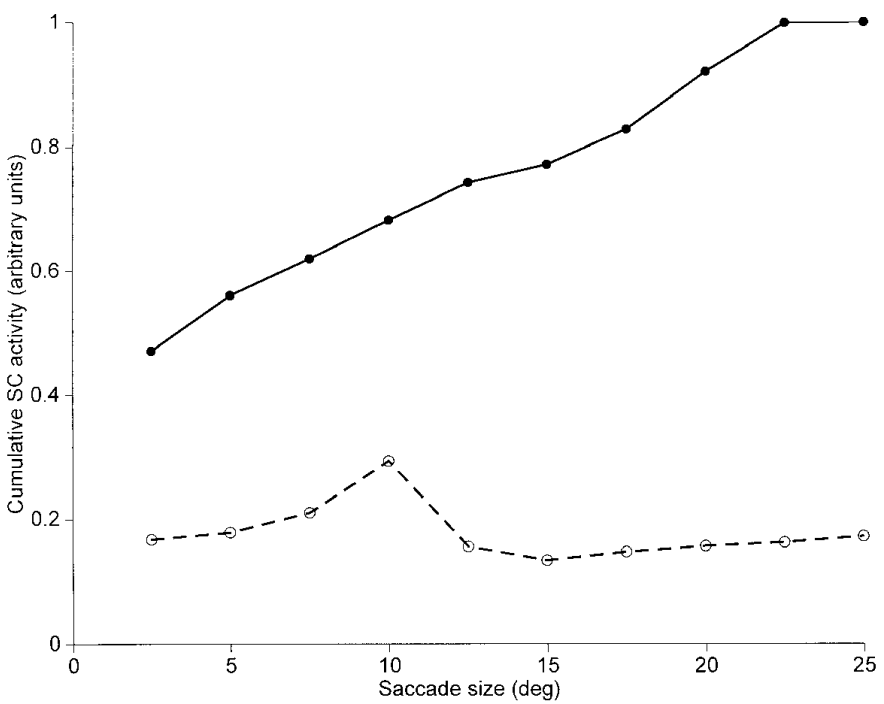

FIG. 16. Total cumulative activity in 2 layers of the SC for a range of horizontal saccade amplitudes. Data points show total activity summed over the contralateral collicular surface and over saccade duration for saccade amplitudes from 2.5 to $25^{\circ}$. Units on the ordinate are normalized to the largest value computed for the buildup layer. $\bullet$ and - , cumulative activity in the buildup layer. $\bigcirc$ and - - - , same measures in the burst layer. input to maintain the discharge of the omnipause neurons and hence the state of fixation in the presaccadic interval. This notion would help explain the marked asymmetry in population activity seen along the rostralcaudal extent of the colliculus before saccade onset.

In our two-dimensional plots, we have chosen to show population activity at saccade end as a natural time of interest but have computed this activity for each 2-ms time point both during the saccade and for $14 \mathrm{~ms}$ after its end. Because of neural delays in the system, collicular events that could control the end of movements would have to precede it. The end of the burst in oculomotor neurons precedes saccade end by $\sim 6 \mathrm{~ms}$ (Keller 1981), and when allowance is made for transmission and synaptic delays, collicular events should lead eye movement events by $\sim 8 \mathrm{~ms}$. This estimate corresponds exactly with the measurements recently made that show a minimum of $8 \mathrm{~ms}$ before eye movements are affected after SC stimulation during ongoing saccades (Miyashita and Hikosaka 1996). The evidence that supports a rostrally directed spread of activity is even less convincing in plots of population activity at $8 \mathrm{~ms}$ before saccade end.

We found rostrally or obliquely directed shifts in the center of mass of the activity in the buildup layer during larger saccades (Fig. 13) as previously reported by Munoz and Wurtz $(1995 \mathrm{~b})$ in their one-dimensional analysis. These shifts were small $(\sim 0.25 \mathrm{~mm})$, and were caused as much by the more rapid collapse of activity during saccades in regions caudal to the center of mass compared with slightly more rostrally situated neurons (as can be seen from careful inspection of Figs. 7, 9, and 11) as by any rostrally directed spread of activity.

Our results are limited to oblique movements not $>45^{\circ}$ from the horizontal meridian because we lacked sufficient coverage of cells located greater than this angle on the colli- 
cular motor map. In addition, oblique saccades can be associated with activity in both colliculi (Wurtz and Goldberg 1972) (see also the cell shown in Fig. 4, right). Many buildup cells are active for saccades in either direction, even for horizontal movements. A more complete analysis would have to include neurons in both colliculi. Finally our analyses are limited to saccades $<25^{\circ}$ in amplitude or even smaller for oblique movements. In the Munoz and Wurtz (1995b) analysis, the apparent rostrally directed spread of activity was most noticeable for larger saccades $\left(30,40\right.$, and $\left.50^{\circ}\right)$. It is possible that we failed to find support for their hypothesis because the largest saccades for which we could estimate population activity were $\sim 20^{\circ}$. However, if a spread of activity is the general mechanism controlling saccades, it should still be present for $20^{\circ}$ movements, which are already "large"' saccades in the natural repertoire of primates (Bahill et al. 1975). In addition, the utility of studying cells at the extreme caudal extent of the colliculus that would be active for eye saccades $>20^{\circ}$ in the fixed-head monkey recently has been questioned by the demonstration that electrical stimulation of the SC produces combined eye-head gaze shifts (Cowie and Robinson 1994; Freedman et al. 1996; Segraves and Goldberg 1991). These studies show that head movement contributions to the evoked gaze shifts become progressively more dominant as the stimulation site is moved more caudal. However, for saccades $<20^{\circ}$ the eye saccade usually makes up the major portion of the gaze shift, if the eyes are centered in the orbit (Freedman et al. 1996; Philips et al. 1995).

\section{Metrics obtained from the two-dimensional analyses}

The two-dimension analyses provide information previously not available from one-dimensional work on horizontal saccades. The activity profiles in the burst layer are nearly symmetrical (when plotted in collicular coordinates) for all sizes and directions of saccades studied within the limits of our data. These two-dimensional plots (Figs. 911 ) and the computations given in Fig. 14 show that McIlwain's point image hypothesis (1976), which states that the active area in the superficial layers of the $\mathrm{SC}$ for a point visual stimulus presented at any eccentricity is constant, is also true for the "movement image"' in the burst layer. The equal areas of activation also confirm the two-dimensional estimates of Ottes et al. (1986) based on more limited data. Based on their one-dimensional analysis, Munoz and Wurtz (1995b) estimated that $\sim 28 \%$ of the collicular surface was active in the burst layer at the peak of activity for any saccade. Similar estimates were made by Ottes et al. (1986) based on their limited spatial sampling of burst cells. Our more extensive two-dimensional analysis shows that the earlier estimates are approximately correct. Our results show that $\sim 25 \%$ of the burst layer is active at saccade onset, and the amount of active area is relatively invariant with saccade size and direction.

In the buildup layer, the size of the active zone increases modestly for horizontal saccades with saccade size for movements $\leq 15^{\circ}$, but overall the active area also remains relatively invariant as a function of saccade vector. The active area in the buildup layer is consistently about twice the size of that in the burst layer.
Figure 14 shows a prominent decline in the peak activity of both burst and buildup neurons as a function of increasing saccade size, which means that the peak of the population activity is lower at more caudal collicular sites coding larger saccades. This trend has not been previously reported and needs further confirmation. It could play an important role in population averaging schemes of collicular function. Lee et al. (1988) note that a true average of a symmetrically arranged population of active burst cells would not yield the movement represented by cells in the geometric center of the population because of the nonlinearity of the amplitude scale on the motor map. They suggest that a nonlinear synaptic weighting of the contribution of cells at different locations to the brain stem saccadic burst generator could solve the problem but the scaling of peak amplitude of cells' discharge as a function of cell location on the SC as seen in our data also could contribute to the solution.

The signals shown in Fig. 15 estimate the summed collicular activity in each layer as a function of time during saccades. If summed activity is assumed to represent SC output, this is an important signal to know because SC output often is hypothesized in single-input/single output (lumped) models to code certain saccadic control variables, e.g., dynamic motor error for the burst layer (Waitzman et al. 1991). In another model, the summed signals from the two layers have been hypothesized to code the desired ocular displacement (i.e., the saccadic goal) for the burst layer and the current ocular displacement in the ongoing saccade in the buildup layer (Optican 1995). It is difficult to reconcile the predictions of the latter theory with the more rapid decline of activity in the burst layer with respect to that in the buildup layer as shown by Fig. 15. In fact, summed buildup activity also declines during the saccade instead of increasing as would be expected if it coded the change in eye position. In yet another model summed output is hypothesized to code saccadic velocity (Scudder 1988).

The composite signals shown in Fig. 15 may represent the total output of the two layers but do not necessarily predict the relative influence of the neurons making up the composite signals on downstream structures. Each neuron in each layer must project its own signal to downstream saccadic control structures, and these distributed inputs may be weighted by location of the projecting cells on the map. In the distributed model of Das et al. (1995), following a learning procedure, weights representing synaptic strength from burst cells increased by a factor of three from rostral to caudal colliculus to produce the larger saccades with higher velocities coded by more caudal regions.

Two approaches have been used to assign the weights for collicular projections to downstream saccadic control structures. In one alternative, they are specified analytically on the basis of the presumed signal being carried by the layer's output (Lefèvre and Galiana 1992; Optican 1995). In an alternative approach, a neural network is trained to produce a candidate set of weights that will solve the dynamic control problem using estimates of the distributed collicular output (Arai et al. 1994; Das et al. 1995; Massone 1994). Although it is not clear at the present time how to best solve or even understand the problem of estimating the distributed connections from population coded structures like the $\mathrm{SC}$, it is true that two-dimensional estimates of the 
spatio-temporal activity in the SC during saccades like those we present here form the essential starting data for such endeavors.

\section{Dynamic control theories of collicular function}

We believe that the dynamic population estimates of collicular activity that we obtained in the present study are compatible with the hypothesis that tonic presaccadic activity of most rostral, ventral neurons in the SC is suppressed before saccade onset for large saccades. The depth of the suppression and its caudal extent both increase with saccade size. Munoz and Wurtz (1995b) posit the existence an organized spread of activity through rostral cells that is driven by a neural mechanism involving the spatial integration of an eye velocity feedback signal by a network of buildup cells (Optican 1995). Thus this hypothesis is an intracollicular theory that controls saccadic dynamics by the present location of the rostrally directed spread of activity until fixation neurons are reactivated, an event that ends the saccade. Instead we argue that the focus of attention for the control of saccade dynamics should be on the discharge of the most active cells (both buildup and burst cells) at the optimal location on the collicular map. Their greatly increased activity at saccade onset and subsequent rapid decline (more rapid in burst neurons than in buildup cells as shown in Fig. 15 ) in activity during the movement could play a role in the control of saccade dynamics similar to that hypothesized for burst cells alone in earlier theories of collicular dynamic control (Das et al. 1995; Waitzman et al. 1991). We view the return of low-level activity in rostral buildup neurons and fixation neurons near or after saccade end as a mechanism assisting the reactivation of omnipause neurons to which both groups are heavily connected (Gandhi and Keller 1997). The return of activity in rostral collicular cells thus would aid in the reestablishment of fixation at the end of a movement. Alternatively, the reactivation of rostral collicular neurons might be associated with the planning of small corrective saccades that normally follow movements made to larger eccentricity targets (Becker 1989). We negated the production of corrective saccades in our experiments by turning off the target before saccade onset but cannot rule out the planing of such movements because our monkeys did make hypometric saccades to the large eccentric target displacements used here.

In contrast to the two dynamic hypotheses just discussed, a third possibility is that the colliculus is not inside a dynamic feedback loop at all. Although it is true that the decline of collicular activity during saccades is correlated with dynamic motor error (Waitzman et al. 1991), correlations do not prove causality. If the colliculus is not inside a dynamic feedback loop that specifically computes dynamic motor error, some other mechanism would be required to reduce collicular burst activity so that it is over (burst cells) or reaches the end of its rapid phase of decline (buildup cells) by saccade end. The decline in discharge (for both types of cells) could be an emergent property of the network of collicular cells being interconnected by near neighbor excitation and more distant lateral inhibition as hypothesized by Arai et al. (1994). In this case, the time constant of decline of activity would be dependent on network connectivity and not the feedback of eye movement information. This possibility is made untenable by the observations made on collicular burst cell discharge during interrupted saccades by Keller and Edelman (1994). Their results showed that burst cell discharge, instead of continuing to decline with its expected time course during the period of interruption when the eyes were not moving, was totally inhibited and then showed a second burst of discharge, the completion of which still remained correlated with the end of the resumed movement. These results are most easily explained by assuming that the colliculus receives dynamic feedback information about eye motion. The results do not prove that dynamic motor error is computed by the feedback loop.

Finally, it is possible that the decline of activity in collicular neurons during saccades is simply imposed on them by input projections from extracollicular sources (Schlag-Rey et al. 1992). In opposition to the latter idea are reports on the temporal properties of either frontal eye field neurons or substantia nigra neurons, the two most likely sources of such extracollicular input, which do not show any discharge that correlates with dynamic motor error (Hikosaka and Wurtz 1983; Segraves and Park 1993).

\section{Classification of cell type}

Although our classification of cell types in the deeper layers of the colliculus agrees qualitatively with the scheme proposed by Munoz and Wurtz (1995a), we used quantitatively different criteria to separate our population of cells into three types for the one-dimensional analyses. In particular, we used the depth of cells from the dorsal surface of the SC as the primary criterion to divide our cells into two layers $(<1.4$ and $>1.6 \mathrm{~mm})$. Cells located in the intermediate zone $(1.5-1.6 \mathrm{~mm}$ from the surface) were classified as buildup or burst cells on the basis of their early presaccadic discharge characteristics.

Similarly there appears to be a continuum in the discharge properties of rostral buildup and fixation neurons. Munoz and Wurtz (1995b) also made a similar observation but then arbitrarily placed a dividing line at a distance of $0.72 \mathrm{~mm}$ from the rostral limit of the SC and classified cells located rostral to this limit, and that paused for saccades, as fixation cells. As an alternative classification, we placed a cell in the buildup class if we could find a small saccades for which the cell showed a saccade-related increase of activity instead of a pause. Because it was difficult to routinely obtain saccades of $<1^{\circ}$ in our monkeys and because the size of the field for which increases in activity were observed decreased with more rostral locations, it is possible that all rostral collicular neurons would have a small region for which they would show increases rather than pauses of activity. In the two-dimensional analyses, we combined both the buildup neurons and the fixation neurons in a single layer in computing the spatio-temporal activity during saccades with our radial basis algorithm. Another recent study also has suggested that "there are no fundamental differences between rostral buildup and fixation neurons" (Krauzlis et al. 1997).

Other classification schemes for intermediate layer collicular cells exist (see Guitton 1991 for a review). Glimcher and Sparks (1992) have described a class of collicular neurons they called prelude bursters that resemble buildup cells 
in the property of extensive presaccadic discharge. However, they have not described the depth of their cells from the surface of the colliculus or whether they show open-field discharge characteristics. In any case, none of our present observations are in conflict with their suggestion that cells with marked presaccadic discharge (their prelude class ) play a role in movement selection. In conclusion, we suggest that the artificial division between fixation neurons and rostral buildup neurons be dropped and instead that they be referred to as tonic rostral collicular neurons. In this new classification scheme, it would be understood that the density of such cells would decline smoothly with caudal progression on the SC.

\section{Location of cells on the colliculus}

There are several ways that one might use to locate recorded cells on the colliculus. Investigators in the past have used metrics based on the motor-related discharge (the movement field) of cells to find a "best'" amplitude and direction for each cell (Munoz and Wurtz 1995b; Ottes et al. 1986). The nonlinear coordinate transformation for the Ottes et al.'s collicular motor map (1986), which is based on stimulation results, then was used to convert each cell's best amplitude and direction coordinates to anatomic location on the colliculus. Because the movement fields of many buildup neurons have very broad spatial peaks or show multiple peaks during saccades, as shown by our present analysis, we believe that using the saccade vector corresponding to the location of the absolute peak discharge over space and time to place cells, is likely to produce large errors (in location) for some neurons. For example, the cell shown in Fig. 5 would be placed at a very caudal location based the preferred saccade for its motor discharge. Yet we knew from the characteristics of other nearby cells recorded on the same perpendicular penetration and from the strong secondary peak in its movement field that it was more likely located at the rostral location where we placed it based on its visual response. Furthermore, some cells showed large movements in the location of their peak discharge over their movement field maps at different perisaccadic time frames.

Therefore, we used the optimal target location of each cell's visual response to generate its best amplitude and direction. Our decision to use the peak of the visual response as the locating criterion for collicular cells was supported by the results of electrical stimulations made on some penetrations. The mean error for buildup cells between the collicular locations computed on the basis of stimulation and that generated by using the peaks of the visual response was about one-half of the mean error that resulted for comparisons between the stimulation implied locations and those computed from the peaks of the movement field fits. Past studies on the correspondence between the visual and motor fields of deeper layer collicular cells found the visual field could be either smaller or larger than the corresponding cell's movement field, but the regions of peak activity in each type of field for a given cell were always congruent (Wurtz and Goldberg 1972). We found, in contrast, that there could be large differences in the location of the visual and motor field peaks for some buildup cells. The apparent discrepancy may be due to sampling differences that appeared to include mostly burst cells in the previous study. Wurtz and Goldberg (1972) also reported that only $60 \%$ of their deeper layer cells showed visual responses in addition to their motor response. We found that $95 \%$ of our cells had a detectable visual response. Perhaps we could better distinguish visual and motor responses with the use of the delayed saccades task as opposed to their use of the regular saccade paradigm.

McIlwain (1976) has pointed out that there may be systematic shifts in the centers of the visual fields of collicular cells in comparison with the center of the retinal locus from which they receive input. This can introduce a systematic bias in placement of the cells on the SC map when the center is defined as the geometric center or the center of mass of the field. We circumvented this potential problem by using the peak of the visual field as our measure of the optimal target vector. In addition, we examined the difference between the location for a cell determined by the center of its visual response and its location determined by electrical microstimulation at the recording site for some of the cells in our sample and did not find a systematic shift in the rostral direction for the use of the visual field centers. Thus it does not seem likely that our use of a different method of localization could have caused us to miss evidence for a rostral spread of activity during saccades.

In conclusion we have described a new technique that may be useful for estimating the spatio-temporal population discharge in topologically organized neural structures. With this technique, we have examined the dynamic activity in two-dimensions and in dorsal and ventral motor layers of the SC during saccades. Our results cast doubt on the validity of the moving wave hypothesis of collicular function. Nevertheless, there are a wide host of limitations in all present studies that sample single cells sequentially over time and in different animals that limit the conclusions that may be drawn from such studies. It seems likely that this problem will not be solved until it is possible to simultaneously sample neurons across the extent of the SC during single saccades.

The authors are grateful to Dr. Lance Optican for helpful discussions on computational issues involved in estimating two-dimensional spatiotemporal activity in the SC.

The research reported here was supported partially by National Institutes of Health Grants R01 EY-06860 and 5T32 GM-08155. Computational aspects of the work was supported partially by IBN-9511365 from the National Science Foundation.

Address for reprint requests: E. L. Keller, Smith-Kettlewell Eye Research Institute, 2232 Webster St., San Francisco, CA 94115.

Received 8 August 1997; accepted in final form 2 April 1998.

\section{REFERENCES}

Anderson, R. W., DAs, S., AND Keller, E. L. Estimation of spatio-temporal neural activity using radial basis function networks. J. Comp. Neurosci. In press.

Arai, K., Keller, E. L., and Edelman, J. A. Two-dimensional neural network model of the primate saccadic system. Neural Networks 7: 11151135, 1994.

Bahill, A. T., Adler, D., And Stark, L. Most naturally occurring human saccades have magnitudes of 15 degrees or less. Invest. Ophthalmol. 14: 468-469, 1975.

BeCKer, W. Metrics of saccades. In: The Neurobiology of Saccadic Eye Movements, edited by R. H. Wurtz and M. E. Goldberg. Amsterdam: Elsevier, 1989, p. 13-67. 
Berthoz, A., Grantyn, A., and Droulez, J. Some collicular efferent neurons code saccadic eye velocity. Neurosci. Lett. 72: 289-294, 1986.

Bruce, C. J. ANd Goldberg, M. E. Primate frontal eye fields. I. Single neurons discharging before saccades. J. Neurophysiol. 53: 603-635, 1985.

ButtNer-EnNever, J. A. AND HoRn, A. K. Neuroanantomy of saccadic omnipause neurons in nucleus raphe interpositus. In: Contempory Ocular Motor and Vestibular Research: A Tribute to David A. Robinson, edited by A. F. Fuchs, T. Brandt, U. Büttner, and D. S. Zee. New York: Georg Thieme Verlag, 1994, p. 488-495.

Cowie, R. J. AND Robinson, D. L. Subcortical contributions to head movements in macaques. I. Contrasting effects of electrical stimulation of a medial pontomedullary region and the superior colliculus. J. Neurophysiol. 72: 2648-2664, 1994.

Crandall, W. F. and Keller, E. L. Visual and oculomotor signals in nucleus reticularis tegmenti pontis in alert monkey. J. Neurophysiol. 54: 1326-1345, 1985.

Das, S., Gandhi, N. J., ANd Keller, E. L. Open-loop simulations of the primate saccadic system using burst cell discharge from the superior colliculus. Biol. Cybern. 73: 509-518, 1995.

De Boor, C. A practical guide to splines. In: Applied Mathematical Sciences, edited by F. John, J. LaSalle, L. Sirovich, and G. Whitham. New York: Springer-Verlag, 1978, p. 235-249.

EFron, B. The Jackknife, the Bootstrap and Other Resampling Plans. Philadelphia: Soc. for Industr. Appl. Math., 1982, p. 75-98.

Freedman, E. G., Stanford, T. R., and Sparks, D. L. Combined eyehead shifts produced by electrical stimulation of the superior colliculus in rhesus monkey. J. Neurophysiol. 76: 927-952, 1996.

GandHI, N. J. AND Keller, E. L. Spatial distribution and discharge characteristics of superior colliculus neurons antidromically activated from the omnipause region in monkey. J. Neurophysiol. 78: 2221-2225, 1997.

Gandhi, N. J., Keller, E. L., AND Hartz, K. E. Interpreting the role of collicular buildup neurons in saccadic eye movement control. Soc. Neurosci. Abstr. 20: 141, 1994.

Glimcher, P. W. AND Sparks, D. L. Movement selection in advance of action in the superior colliculus. Nature 355: 542-545, 1992.

GuitTon, D. Control of saccadic eye and gaze movements by the superior colliculus and basal ganglia. In: Eye Movements, edited by R.H.S. Carpenter. Boca Raton, FL: CRC, 1991, p. 244-276.

HiKosaK A, O. AND WuRTZ, R. H. Visual and oculomotor functions of monkey substantia nigra pars reticulata. IV. Relationship of substantia nigra to superior colliculus. J. Neurophysiol. 49: 1285-1301, 1983.

Hoel, P. G. Introduction to Mathematical Statistics. New York: John Wiley, 1965 , chapt. 13 , p. $335-341$.

Judge, S. J., Richmond, B. J., And Chu, F. C. Implantation of magnetic search coils for measurement of eye position: an improved method. Vision Res. 20: 535-538, 1980.

KelLeR, E. L. Oculomotor neuron behavior. In: Models of Oculomotor Behavior and Control, edited by B. L. Zuber. Boca Raton, FL: CRC, 1981, p. 1-19.

Keller, E. L. AND EDELman, J. A. Use of interrupted saccade paradigm to study spatial and temporal dynamics of saccadic burst cells in superior colliculus in monkey. J. Neurophysiol. 72: 2754-2770, 1994.

Kooperberg, C., Stone, C. J., And Truong, Y. K. Hazard regression. J. Am. Stat. Assoc. 90: 77-94, 1995.

Krauzlis, R. J., Basso, M. A., AND Wurtz, R. H. Shared motor error for multiple eye movements. Science 276: 1693-1695, 1997.

LeE, C., Rohrer, W. H., AND Sparks, D. L. Population coding of saccadic eye movements by neurons in the superior colliculus. Nature 332: $357-$ 360,1988 .

Lefèvre, P. And Galiana, H. L. Dynamic feedback to the superior colliculus in a neural network model of the gaze control system. Neural Networks 5: 871-890, 1992.

MARQUARDT, D. An algorithm for least-squares estimation of non-linear parameters. SIAM J. Appl. Math. 11: 431-441, 1963.

MASsONE, L. E. A neural-network system for control of eye movements: basic mechanisms. Biol. Cybern. 71: 293-305, 1994.

McIlwaIN, J. T. Large receptive fields and spatial transformations in the visual system. In: Neurophysiology II, edited by R. Porter. Baltimore: University Park Press, 1976, vol. 10, p. 224-242.

Miyashita, N. AND Hikosaka, O. Minimal synaptic delay in the saccadic output pathway of the superior colliculus studied in awake monkey. Exp. Brain Res. 112: 187-196, 1996.

Moody, J. AND DARKEN, C. J. Fast learning in networks of locally-tuned processing units. Neural Comput. 1: 281-294, 1989.

Munoz, D. P., Guitton, D., AND Pélisson, D. Control of orienting gaze shifts by tecto-reticulo-spinal system in the head-free cat. III. Spatiotemporal characteristics of phasic motor discharges. J. Neurophysiol. 66: 1642-1666, 1991a.

Munoz, D. P., PÉlisson, D., AND GuitTon, D. Movement of neural activity on the superior colliculus motor map during gaze shifts. Science 251: 1358-1360, $1991 \mathrm{~b}$.

MunOz, D. P. AND WuRTZ, R. H. Fixation cells in monkey superior colliculus. I. Characteristics of cell discharge. J. Neurophysiol. 70: 559-575, 1993.

MunOz, D. P. AND WuRTZ, R. H. Saccade-related activity in monkey superior colliculus. I. Characteristics of burst and buildup cells. J. Neurophysiol. 73: 2313-2333, 1995a.

MunOz, D. P. AND WuRTZ, R. H. Saccade-related activity in monkey superior colliculus. II. Spread of activity during saccades. J. Neurophysiol. 73: 2334-2348, $1995 \mathrm{~b}$.

OpticAN, L. M. A field theory of saccade generation: temporal-to-spatial transform in the superior colliculus. Vision Res. 35: 3313-3320, 1995.

OtTes, F. P., Van Gisbergen, J.A.M., And EgGermont, J. J. Visuomotor fields of the superior colliculus: a quantitative model. Vision Res. 26: 857-873, 1986.

Phillips, J., Ling, L., Fuchs, A., Siebold, C., And Plorde, J. Rapid horizontal gaze movement in the monkey. J. Neurophysiol. 73: 1632 $1652,1995$.

Richmond, B. AND Optican, L. M. Temporal encoding of two-dimensional patterns by single units in primate inferior temporal cortex. II. Quantification of response waveform. J. Neurophysiol. 57: 147-161, 1987.

Robinson, D. A. A method of measuring eye movement using a scleral search coil in a magnetic field. IEEE Trans. Bio-Med. Eng. 10: 137145, 1963.

Robinson, D. A. Eye movements evoked by collicular stimulation in the alert monkey. Vision Res. 12: 1795-1808, 1972.

RoBinson, D. A. Oculomotor control signals. In: Basic Mechanisms of Ocular Motility and Their Clinical Implications, edited by G. Lennerstrand and P. Bach-Y-Rita. Oxford: Pergamon, 1975, p. 337-374.

Schlag-Rey, M., Schlag, J., and Dassonville, P. How the frontal eye field can impose a saccade goal on superior colliculus neurons. J. Neurophysiol. 67: 1003-1005, 1992.

SCUDDER, C. A. A new local feedback model of the saccadic burst generator. J. Neurophysiol. 59: 1455-1475, 1988.

Segraves, M. A. AND Goldberg, M. E. Properties of eye and head movements evoked by electrical stimulation of the monkey superior colliculus. In: The Head-Neck Sensory-Motor System, edited by A. Berthoz, W. Graf, and P. Vidal. Oxford: Oxford Univ. Press, 1991, p. 321-328.

Segraves, M. A. AND Park, K. The relationship of monkey frontal eye field activity to saccade dynamics. J. Neurophysiol. 69: 1880-1889, 1993.

Sparks, D. L. AND Hartwich-Young, R. The deep layers of the superior colliculus. In: The Neurobiology of Saccadic Eye Movements, Reviews of Oculomotor Research, edited by R. Wurtz and M. Goldberg. Amsterdam: Elsevier, 1989, vol. 3, p. 213-255.

Van Gisbergen, J.A.M., Van Opstal, A. J., and TaX, A. A. Collicular ensemble coding of saccades based on vector summation. Neuroscience 21: $541-555,1987$.

VAn Opstal, A. J. AND VAn Gisbergen, J.A.M. A model for collicular efferent mechanisms underlying the generation of saccades. Brain Behav Evol. 33: 90-94, 1989.

Waitzman, D. M., Ma, T. P., Optican, L. M., AND WuRTZ, R. H. Superior colliculus neurons mediate the dynamic characteristics of saccades. $J$. Neurophysiol. 66: 1716-1737, 1991.

Walpole, R. E. AND Myers, R. H. Probability and Statistics for Engineers and Scientists. New York: MacMillan, 1989, chapt. 10, p. 635-639.

WURTZ, R. H. Vision for the control of movement. The Friedenwald Lecture. Invest. Ophthalmol. Vis. Sci. 37: 2130-2145, 1996.

Wurtz, R. H. AND Goldberg, M. E. Activity of superior colliculus in behaving monkey. III. Cells discharging before eye movements. J. Neurophysiol. 35: 575-586, 1972. 